\title{
Restrictions for $n$-point vertices in higher-spin theories
}

\author{
Stefan Fredenhagen, ${ }^{a, b}$ Olaf Krüger $^{a}$ and Karapet Mkrtchyan ${ }^{c}$ \\ ${ }^{a}$ University of Vienna, Faculty of Physics, \\ Boltzmanngasse 5, 1090 Vienna, Austria \\ ${ }^{b}$ Erwin Schrödinger International Institute for Mathematics and Physics, \\ Boltzmanngasse 9, 1090 Vienna, Austria \\ ${ }^{c}$ Scuola Normale Superiore and INFN, \\ Piazza dei Cavalieri 7, 56126 Pisa, Italy \\ E-mail: stefan.fredenhagen@univie.ac.at, olaf.krueger@univie.ac.at, \\ karapet.mkrtchyan@sns.it
}

AbSTRACT: We give a simple classification of the independent $n$-point interaction vertices for bosonic higher-spin gauge fields in $d$-dimensional Minkowski spacetimes. We first give a characterisation of such vertices for large dimensions, $d \geq 2 n-1$, where one does not have to consider Schouten identities due to over-antisymmetrisation of spacetime indices. When the dimension is lowered, such identities have to be considered, but their appearance only leads to equivalences of large- $d$ vertices and does not lead to new types of vertices. We consider the case of low dimensions $(d<n)$ in detail, where a large number of Schouten identities leads to strong restrictions on independent vertices. We also comment on the generalisation of our results to the intermediate region $n \leq d \leq 2 n-2$. In all cases, the independent vertices are expressed in terms of elementary manifestly gauge-invariant quantities, suggesting that no deformations of the gauge transformations are induced.

KeYwords: Higher Spin Gravity, Scattering Amplitudes, Higher Spin Symmetry, SpaceTime Symmetries

ARXIV EPRINT: 1912.13476 


\section{Contents}

1 Introduction 1

2 Preliminaries $\quad 4$

2.1 Vertex generating operators 4

2.2 Equivalence relations for vertex generating operators 5

2.3 Imposing gauge invariance 8

3 The case $2 n-1 \leq d \quad 9$

3.1 Gauge invariants 9

$\begin{array}{ll}3.2 \text { Building blocks of vertices } & 11\end{array}$

4 Lower dimension: dealing with Schouten identities 13

$5 \quad$ The case $n>d \quad 15$

$\begin{array}{lll}\text { 5.1 A minimal generating set of Schouten identities } & 16\end{array}$

$\begin{array}{ll}5.2 \text { The choice of representative } & 17\end{array}$

$\begin{array}{lll}5.3 \text { General restrictions from gauge invariance } & 19\end{array}$

$\begin{array}{ll}5.4 \text { Restrictions for } \mathcal{V} & 19\end{array}$

$\begin{array}{lll}5.5 & \text { Proofs } & 20\end{array}$

5.5.1 Proof of eq. (5.4) 20
5.5.2

5.5.2 Proof of eq. (5.7) 22

6 Parity-odd vertices $\quad 25$

$\begin{array}{llr}7 & \text { Discussion } & 27\end{array}$

\section{Introduction}

In this paper, we investigate a Lagrangian formulation of higher-spin (HS) theories in arbitrary dimensions. The aim of this work is, in particular, to obtain restrictions for all possible independent interaction vertices of order $n \geq 4$ for massless higher-spin fields, extending the three-dimensional results of [1]. Together with the earlier results on the cubic vertices [2-9] (see also [10-15]), this work intends to complete the classification of all independent interacting deformations of free massless HS Lagrangians [16, 17] to the lowest order in the deformation parameters (coupling constants) in Minkowski spacetime of arbitrary dimensions $d \geq 3$.

HS Gravities [18-20] (see, e.g., [21, 22] for reviews) are generalisations of Einstein's General Relativity which involve higher-spin gauge fields. These are symmetric tensor 
(Fronsdal) fields ${ }^{1} \phi_{\mu_{1} \ldots \mu_{s}}$, described by the Fronsdal action [16] at free level, describing massless particles of spin $s$ upon quantisation. ${ }^{2}$ A set of free HS fields can be described by a Lagrangian which is a sum of Fronsdal Lagrangians for spin $s$ fields. However, a full non-linear Lagrangian of interacting Fronsdal fields is not available to date.

Such theories are strongly constrained by gauge invariance, necessary for consistency. These gauge transformations extend those of General Relativity - spacetime reparametrisations, or diffeomorphisms - to larger symmetries, involving gauge parameters that are Lorentz tensors of rank $(s-1)$ for each massless spin $s$ field. This extension of symmetries can potentially resolve some problems of General Relativity (singularities, quantisation problem, etc.), making HS Gravity an attractive field of investigation.

The corresponding gauge transformation for free fields reads ${ }^{3}$

$$
\delta^{(0)} \phi_{\mu_{1} \ldots \mu_{s}}=s \partial_{\left(\mu_{1}\right.} \epsilon_{\left.\mu_{2} \ldots \mu_{s}\right)},
$$

which generalises the well known expressions for massless vector fields $(s=1)$ in gauge theory and the Graviton $(s=2)$ in linearised gravity theory.

The naive intuition from lower-spin model building suggests that one can pick an arbitrary collection of fields, including massless HS fields, and the gauge symmetries will partly constrain the interactions, leaving room for a large parameter space of theories. It turns out that the severe constraints from HS gauge invariance rule out theories with an arbitrary choice of the particle content. Therefore, one is easily led to negative results if one chooses an arbitrary starting setup for constructing a theory with massless HS spectrum. This striking difference from textbook examples makes it tempting to conclude (after some attempts) that such theories cannot exist.

The problem can be traced to the global symmetries of the theory (see, e.g., [23]). One can construct a HS theory by looking for suitable global symmetry algebras, which have to satisfy the so-called admissibility condition [24]. In this way, infinitely many potential candidate algebras are ruled out (see, e.g., [25, 26]). This is crucial in deriving a list of admissible HS algebras [27, 28] and constructing full non-linear HS equations [18-20] in the frame formulation, proving the existence of a theory with massless HS fields. The resulting theory, however, has unusual properties: there is an infinite tower of massless higher-spin fields with $s=0,1,2, \ldots$ and a necessarily non-zero cosmological constant [29]. The need for a non-zero cosmological constant is related to diffeomorphism transformations, as explained in [30, 31], essential for the Fradkin-Vasiliev solution to the Aragone-Deser problem [32]. This argument, together with the holographic conjectures (see, e.g., [33, 34]) motivated the intense studies of HS interactions, especially in (Anti-)de Sitter $((A) d S)$ background [35-50].

The frame-like formulation of HS gravities that led to successful developments (including Vasiliev's non-linear equations [18-20] and their recent generalisations [51, 52]) registered less progress so far in understanding the corresponding Lagrangian formulation. On the other hand, the metric-like formulation $[16,17,48]$ is a simple suitable setup for

\footnotetext{
${ }^{1}$ The index $s$ is the spin of the field and $\mu_{i}=0, \ldots, d-1$ in $d$ dimensions.

${ }^{2}$ In this paper, we will restrict ourselves to integer-spin (bosonic) fields for simplicity.

${ }^{3}$ The round (square) brackets denote (anti-)symmetrisation with weight one.
} 
classifying interaction vertices and deriving restrictions on interacting Lagrangians. Here, we work in the framework of the Noether-Fronsdal program (see [53-68] for related literature and [9] for a recent summary of the status of the problem) to classify independent vertices of order $n \geq 4$ in arbitrary dimensions $d \geq 3$, generalising the $d=3$ results obtained earlier in [1].

The situation is different only in three dimensions, where the interacting HS theories can admit arbitrary Einstein backgrounds (including Minkowski) as well as a finite spectrum of massless HS fields (see, e.g., [69-74]). However, such massless HS fields do not correspond to propagating particles in $d=3$, while the inclusion of matter leads to a situation similar to the higher-dimensional story in many ways.

It is also interesting to note here, that four dimensions is also somewhat special: there, the Aragone-Deser problem is relevant at the Lagrangian level for descriptions using Fronsdal fields, while the minimal coupling to gravity is available at the level of amplitudes (see, e.g., $[7,75-78])$ and light-cone vertices $[2-4,79-82]$ together with other vertices that are absent in spacetime dimensions $d \geq 5$. Most importantly, these vertices seem to be essential for the consistency of the non-linear theory $[3,4]$ in four dimensions.

The Noether-Fronsdal program is a systematic approach to perturbatively construct a Lagrangian $\mathcal{L}$ for an arbitrary interacting HS theory order by order. In this procedure, $\mathcal{L}$ is expanded in powers of small parameters $g_{n}$,

$$
\mathcal{L}=\mathcal{L}_{2}+\sum_{n \geq 3} g_{n} \mathcal{L}_{n}+O\left(g_{n}^{2}\right)
$$

Here, $\mathcal{L}_{2}$ denotes the free Fronsdal Lagrangian and another sum over the different kinds of $n$-point vertices $\mathcal{L}_{n}$ is suppressed.

The action must be gauge-invariant, hence, $\delta \mathcal{L}$ equals a total derivative, where $\delta$ is obtained by a deformation of the free gauge transformation $\delta^{(0)}$,

$$
\delta=\delta^{(0)}+\sum_{k \geq 1} \delta^{(k)}
$$

Here, the deformation $\delta^{(k)}$ is of $k$-th order in the fields. Since our aim is to find constraints for the independent vertex structures (i.e. linear in the coupling constants ${ }^{4}$ ), the $n$-point vertex must satisfy

$$
\delta^{(0)} \mathcal{L}_{n}+\delta^{(n-2)} \mathcal{L}_{2}=0 \text { up to total derivatives . }
$$

In this paper, we find restrictions for all independent $n$-point vertices $\mathcal{L}_{n}$ for massless HS fields in arbitrary dimension $d \geq 3$, such that they satisfy eq. (1.3). ${ }^{5}$ From that, we deduce a simple classification of vertices. For a summary of the explicit results, see the beginning of section 7 .

\footnotetext{
${ }^{4}$ Gauge invariance provides constraints to fix the terms proportional to higher powers of coupling constants. We are interested here in the structures that parametrise the non-trivial deformations at the lowest order in the coupling constants.

${ }^{5}$ We consider a flat Minkowski spacetime but comment also on $(A) d S$ backgrounds in section 7 .
} 
The paper is organised as follows: in section 2, we set up notations and provide the mathematical framework for our analysis. There, we discuss that we have to analyse three different cases separately: large dimensions $d \geq 2 n-1$ (see section 3), low dimensions $d<n$ (see section 5) and the intermediate case (see section 4 and comments in section 7). We mostly consider parity-even vertices, but give a generalisation to parity-odd vertices in section 6 . We finally conclude in section 7 .

\section{Preliminaries}

We want to constrain the $n$-point independent vertices $\mathcal{L}_{n}$ that may constitute the lowest order deformations of the free Lagrangian for massless HS fields. For this purpose, we restrict ourselves to the traceless and transverse (TT) sector of the Lagrangian as in [1]. We refer the reader to a more detailed discussion ${ }^{6}$ on the TT vs off-shell vertices in [48]. In the following, we therefore assume that the tensors $\phi_{\mu_{1} \ldots \mu_{s}}$ that describe the gauge fields, are traceless, divergence-free and the corresponding free equation of motion is given by the (massless) Klein-Gordon equation, hence

$$
g^{\mu_{1} \mu_{2}} \phi_{\mu_{1} \ldots \mu_{s}}=0, \quad \partial^{\mu_{1}} \phi_{\mu_{1} \ldots \mu_{s}}=0,\left.\quad \partial^{\nu} \partial_{\nu} \phi_{\mu_{1} \ldots \mu_{s}}\right|_{\text {free e.o.m. }}=0 .
$$

The relaxation of these conditions will allow to reconstruct the full off-shell counterpart of the $\mathrm{TT}$ vertices as in $[6,48]$.

\subsection{Vertex generating operators}

It is very convenient to contract the indices of the fields each with an auxiliary vector variable $a^{\mu}$,

$$
\phi^{(s)}(x, a)=\frac{1}{s !} \phi_{\mu_{1} \ldots \mu_{s}}(x) a^{\mu_{1}} \cdots a^{\mu_{s}} .
$$

This has several advantages: first, we do not have to tackle expressions with too many indices and secondly, the tensor $\phi_{\mu_{1} \cdots \mu_{s}}$ is by construction symmetric. We will also note later on that the complexity of index contractions will be reduced a lot. For example, using the short-hand notation $P^{\mu}=\partial_{x^{\mu}}$ and $A^{\mu}=\partial_{a^{\mu}}$, the relations in eq. (2.1) simplify to

$$
A^{2} \phi^{(s)}=0, \quad A \cdot P \phi^{(s)}=0,\left.\quad P^{2} \phi^{(s)}\right|_{\text {free e.o.m. }}=0 .
$$

We call these relations collectively Fierz equations [83].

Now, each $n$-point vertex $\mathcal{L}_{n}$ in eq. (1.2) is a product of $n$ massless bosonic fields (and possibly derivatives thereof). But it has to be a Lorentz scalar, hence, all indices of the fields (and of the derivatives) must be fully contracted. For now, let us concentrate on parity-even vertices - we consider parity-odd vertices in section 6 . Then, we can write $\mathcal{L}_{n}$ in the following, very convenient way:

$$
\mathcal{L}_{n}(x)=\left.\mathcal{V}\left(\prod_{i=1}^{n} \phi_{i}\left(x_{i}, a_{i}\right)\right)\right|_{\substack{x_{i}=x \\ a_{i}=0}} .
$$

\footnotetext{
${ }^{6}$ For massive fields, the restriction to TT is a choice of field redefinition freedom and therefore completely legitimate (as discussed in appendix B.1 of [26]), while for massless fields it can introduce subtleties in some formulations, as discussed in section 4.5 of [48].
} 
This needs some explanation:

- We use the notation set up in eq. (2.2) and drop the spin labels of the fields: $\phi_{i}$ is a spin $s_{i}$ field, $\phi_{i}=\phi^{\left(s_{i}\right)}$.

- The term in brackets represents a function of the spacetime coordinates $x_{i}$ and the auxiliary vector variables $a_{i}$. The vertex generating operator $\mathcal{V}$ performs the index contractions between the fields $\phi_{i}$ as follows: let $P_{i}^{\mu}=\partial_{x_{i}^{\mu}}$ and $A_{i}^{\mu}=\partial_{a_{i}^{\mu}}$ as in eq. (2.3). Then, $\mathcal{V}$ must be a polynomial in the following commuting variables:

$$
z_{i j}=\left.A_{i} \cdot A_{j}\right|_{1 \leq i \leq j \leq n}, \quad y_{i j}=\left.A_{i} \cdot P_{j}\right|_{1 \leq i, j \leq n}, \quad s_{i j}=\left.P_{i} \cdot P_{j}\right|_{1 \leq i \leq j \leq n} .
$$

The operator $z_{i j}$ induces a single contraction of indices between the fields $\phi_{i}$ and $\phi_{j}$, whereas $y_{i j}$ will take one index of the field $\phi_{i}$ and contract it with a derivative which acts on the field $\phi_{j}$. Finally, the operators $s_{i j}$ will introduce extra derivatives (a derivative of $\phi_{i}$ is contracted with a derivative of $\phi_{j}$ ); these are called Mandelstam variables.

- Since all of the indices in $\mathcal{L}_{n}$ have to be contracted, we discard all terms that still contain at least one of the auxiliary variables when $\mathcal{V}$ acted on the terms in brackets. Thus, we set $a_{i}=0$ in the end, which ensures that $\mathcal{L}_{n}$ is Lorentz invariant. Finally, we also set $x_{i}=x$. The splitting of the coordinates is useful to keep track of the derivatives acting on different fields, and has no physical consequences.

All in all, we translated the problem of "what is the most general form of the parityeven $n$-point vertex $\mathcal{L}_{n}$ ' to the question 'what is the most general form of the vertex generating operator $\mathcal{V}$ in the polynomial ring $\mathbb{R}\left[y_{i j},\left.z_{i j}\right|_{i \leq j},\left.s_{i j}\right|_{i \leq j}\right]$ '. The connection between Lagrangian $\mathcal{L}_{n}$ and operator $\mathcal{V}$ is given by eq. (2.4). We also ensured that $\mathcal{L}_{n}$ is Lorentz invariant.

There are two questions arising now. First of all, there are equivalence relations for Lagrangians: e.g., two Lagrangians that differ by a total derivative lead to the same action. We call them equivalent in this case. What does this imply for the corresponding vertex generating operators? Secondly, how do we have to constrain $\mathcal{V}$ such that $\mathcal{L}_{n}$ is gauge invariant? We present a general answer to these questions in the remainder of this section and give more details in sections 3 and 5 .

\subsection{Equivalence relations for vertex generating operators}

We must take into account that different Lagrangians may describe the same theory. We say that they are equivalent in this case and evidently, we are only interested in $\mathcal{L}_{n}$ up to equivalence. When we encode the Lagrangians via vertex generating operators, we need to introduce a notion of equivalence for operators: vertex operators $\mathcal{V}$ and $\mathcal{V}^{\prime}$ are equivalent, $\mathcal{V} \approx \mathcal{V}^{\prime}$, iff the two Lagrangians $\mathcal{L}_{n}$ and $\mathcal{L}_{n}^{\prime}$, constructed from them via eq. (2.4) are also equivalent. We are hence only interested in $\mathcal{V}$ up to equivalence and summarise the different kinds of equivalence relations in the following.

The first kind of equivalence relations arises from field redefinitions $\phi_{i} \mapsto \phi_{i}+\delta \phi_{i}$, where $\delta \phi_{i}$ is non-linear in the fields. These do not change the theory, but affect the 
Lagrangian. For example, terms in $\mathcal{L}_{2}$ may contribute to $\mathcal{L}_{n}$ when the fields are redefined non-linearly. But in this way, the $n$-point vertices only change by terms that vanish when the free equations of motion are imposed. We say that two Lagrangians are equivalent, when they are related by such field redefinitions and deduce from eq. (2.4) that we can choose $\mathcal{V}$ to be independent of $s_{i i}$. Furthermore, we assume that $\mathcal{V}$ does not depend on $z_{i i}$ and $y_{i i}$ because the fields are traceless and divergence-free.

Mathematically speaking, we impose the equivalence relations

$$
y_{i i} \approx 0, \quad z_{i i} \approx 0, \quad s_{i i} \approx 0
$$

and deduce that each operator in the ideal $\left\langle y_{i i}, z_{i i}, s_{i i}\right\rangle \subset \mathbb{R}\left[y_{i j},\left.z_{i j}\right|_{i \leq j},\left.s_{i j}\right|_{i \leq j}\right]$ is equivalent to 0 . Hence, we can construct equivalence classes of vertex generating operators,

$$
[\mathcal{V}] \in \frac{\mathbb{R}\left[y_{i j},\left.z_{i j}\right|_{i \leq j},\left.s_{i j}\right|_{i \leq j}\right]}{\left\langle y_{i i}, z_{i i}, s_{i i}\right\rangle} .
$$

The quotient ring is isomorphic to the subring $\mathcal{R}=\mathbb{R}\left[\left.y_{i j}\right|_{i \neq j},\left.z_{i j}\right|_{i<j},\left.s_{i j}\right|_{i<j}\right]$,

$$
\frac{\mathbb{R}\left[y_{i j},\left.z_{i j}\right|_{i \leq j},\left.s_{i j}\right|_{i \leq j}\right]}{\left\langle y_{i i}, z_{i i}, s_{i i}\right\rangle} \simeq \mathcal{R} \subset \mathbb{R}\left[y_{i j},\left.z_{i j}\right|_{i \leq j},\left.s_{i j}\right|_{i \leq j}\right],
$$

so we can choose the vertex generating operator as $\mathcal{V} \in \mathcal{R}$. In other words, we simply dropped the dependence of $\mathcal{V}$ on $y_{i i}, z_{i i}$ and $s_{i i}$.

Secondly, acting with the operator $D^{\mu}=\sum_{j=1}^{n} P_{j}^{\mu}$ on the term in brackets in eq. (2.4) gives a total derivative in the Lagrangian. This does not change the action and hence, does not affect the theory. Therefore, we impose the equivalence relations

$$
A_{i} \cdot D=\sum_{j=1}^{n} y_{i j} \approx 0, \quad P_{i} \cdot D=\sum_{j=1}^{n} s_{i j} \approx 0 .
$$

These together generate an ideal $\mathcal{I}_{D} \subset \mathcal{R}$ and in the following, we consider equivalence classes of vertex generating operators in the quotient ring

$$
[\mathcal{V}] \in \frac{\mathcal{R}}{\mathcal{I}_{D}} .
$$

As for the equivalence relations in eq. (2.6), we could choose a convenient representative $\mathcal{V}$ in $\mathcal{R}$, but it turns out to be better to keep the quotient ring structure for now.

A last equivalence stems from 'Schouten identities', i.e. relations following from overantisymmetrisation of spacetime indices. These spacetime dimension-dependent identities are exact relations at the Lagrangian level. In the polynomial ring $\mathcal{R}$, however, we forgot that we work in $d$ dimensions. Therefore, we have to impose Schouten identities as equivalence relations for vertex generating operators, ${ }^{7}$ which form an ideal $\mathcal{I}_{S} \subset \mathcal{R}$ as follows: let

\footnotetext{
${ }^{7}$ Formally, let $\iota_{d}$ be the map

$$
\iota_{d}: \quad \mathcal{R} \quad \rightarrow \quad \mathbb{R}\left[P_{i}^{\mu}, A_{i}^{\mu}\right]
$$$$
\mathcal{V}\left(z_{i j}, y_{i j}, s_{i j}\right) \mapsto \mathcal{V}\left(A_{i} \cdot A_{j}, A_{i} \cdot P_{j}, P_{i} \cdot P_{j}\right)
$$

that replaces the operators $z_{i j}, y_{i j}$ and $s_{i j}$ by their definitions in eq. (2.5). $\iota_{d}$ therefore reintroduces the operators $P_{i}$ and $A_{i}$ and hence, spacetime indices in $d$ dimensions in the vertex generating operator $\mathcal{V}$. The kernel $\iota_{d}^{-1}(0)$ of this map is what we call the ideal of Schouten identities in $d$ dimensions.
} 
$b=\left(P_{1}, \ldots, P_{n}, A_{1}, \ldots A_{n}\right)$ be a vector of derivative operators and consider the symmetric $2 n \times 2 n$ matrix

$$
\mathcal{B}=\left.\left(b_{K} \cdot b_{L}\right)\right|_{K, L \in(1, \ldots, 2 n)}=\left(\begin{array}{cc}
\mathcal{S} & \mathcal{Y}^{T} \\
\mathcal{Y} & \mathcal{Z}
\end{array}\right) .
$$

Here, $\mathcal{S}=\left(s_{i j}\right), \mathcal{Y}=\left(y_{i j}\right), \mathcal{Z}=\left(z_{i j}\right)$ are symmetric $(n \times n)$-matrices with elements in $\mathcal{R}$. With the equivalence relations in eq. (2.6), the diagonal elements of $\mathcal{S}, \mathcal{Y}$ and $\mathcal{Z}$ vanish equivalently. We also keep in mind that there are further equivalence relations from eq. (2.7) which introduce a linear relation among the first $n$ rows (and columns) of $\mathcal{B}$, but we do not apply them right now.

Then, the ideal $\mathcal{I}_{S}$ is generated by all $(d+1) \times(d+1)$ minors of $\mathcal{B}$. We show this in a moment, but note first that this implies that $\mathcal{I}_{S}$ is trivial for $d \geq 2 n-1$. Indeed, in this case, there is only one such minor, namely when equality holds. This minor is $\operatorname{det} \mathcal{B}$, which is equivalent to zero due to the equivalence relations in $\mathcal{I}_{D}$ (the first $n$ rows add up to a total derivative). Now we show that for $d<2 n-1$, the above statement is true. Indeed, remove $(2 n-d-1)$ rows and columns from $\mathcal{B}$, such that only the rows $K_{1}, \ldots, K_{d+1} \in(1, \ldots, 2 n)$ and the columns $L_{1}, \ldots, L_{d+1} \in(1, \ldots, 2 n)$ remain and call the resulting $(d+1) \times(d+1)$-matrix $M$. Then,

$$
\begin{aligned}
\operatorname{det} M & =\delta_{\nu_{1}}^{\mu_{1}} \cdots \delta_{\nu_{d+1}}^{\mu_{d+1}} B_{\mu_{1} K_{1}} \cdots B_{\mu_{d+1} K_{d+1}} B_{\left[L_{1}\right.}^{\nu_{1}} \cdots B_{\left.L_{d+1}\right]}^{\nu_{d+1}} \\
& =\delta_{\nu_{1} \cdots \nu_{d+1}}^{\mu_{1} \cdots \mu_{d+1}} B_{\mu_{1} K_{1}} \cdots B_{\mu_{d+1} K_{d+1}} B_{L_{1}}^{\nu_{1}} \cdots B_{L_{d+1}}^{\nu_{d+1}}
\end{aligned}
$$

and acting with it on the term in brackets in eq. (2.4) yields a term in the Lagrangian with over-antisymmetrised indices. On the other hand, each term in the Lagrangian with over-antisymmetrised indices corresponds to a vertex generating operator $\mathcal{V}$ that contains a factor of the form on the r.h.s. of eq. (2.9) for a certain set of indices $K_{i}, L_{i} \in(1, \ldots, 2 n)$. Hence, $\mathcal{V} \in \mathcal{I}_{S}$.

At this step, it is convenient to introduce the notion of the level of a Schouten identity. To this end, let us first define the level of the rows and columns of $\mathcal{B}$ as follows: the first $n$ rows and columns of $\mathcal{B}$ are of level 0 and all others are of level 1 . Furthermore, each $(d+1) \times(d+1)$-submatrix $M$ of $\mathcal{B}$ that is obtained by removing rows and columns inherits those row and column levels from $\mathcal{B}$. Then, the sum of row and column levels of $M$ equals the power of $A_{i}^{\mu}$ operators in $\iota_{d}(\operatorname{det} M)$. This is what we call the level of the Schouten identity $\operatorname{det} M \approx 0$. Denote by $I(k)$ the ideal generated by all Schouten identities of level $k$, then we have

$$
\mathcal{I}_{S}=\sum_{k=0}^{2 d+2} I(k)
$$

where again, $d$ denotes the spacetime dimension.

Now, we consider three cases:

- For large dimensions, $d \geq 2 n-1$, as discussed before, there are no non-trivial Schouten identities at all (the only possible Schouten identities arise in the case $d=2 n-1$, but they are zero up to total derivatives, so they are already contained in $\mathcal{I}_{D}$ ). This case is much simpler and we treat it separately in section 3 . 
- For large values of $n, d<n$, only the subideal $I(0)$ might be trivial (namely for $d+1=n$, where the level 0 Schouten identities vanish up to a total derivative and thus are already contained in $\mathcal{I}_{D}$ ). Thanks to the variety of Schouten identities available, we are able to perform a lot of simplifications. We treat this case in section 5 .

- In the intermediate case $2 n-2 \geq d \geq n$ only the ideals of level $2 d-2 n+4, \ldots, 2 n$ are non-trivial. We will not study this case in full detail here, but a general characterisation of the corresponding vertices is given in section 7 .

All in all, we have now considered all possible equivalences for parity-even Lagrangians. Because of the freedom of field redefinitions, we consider $\mathcal{V} \in \mathcal{R}$ and we divide out the ideals generated by total derivatives $\left(\mathcal{I}_{D}\right)$ and Schouten identities $\left(\mathcal{I}_{S}\right)$,

$$
[\mathcal{V}] \in \frac{\mathcal{R}}{\mathcal{I}}, \quad \mathcal{I}=\mathcal{I}_{S}+\mathcal{I}_{D}
$$

\subsection{Imposing gauge invariance}

Finally, we require that $\mathcal{L}$ is gauge invariant, i.e. that it satifies eq. (1.3). What does this imply for the corresponding vertex generating operator $\mathcal{V}$ ? Note first that the second term in eq. (1.3) vanishes when the free equations of motions are imposed. In other words, the requirement of gauge invariance for the independent vertex structures reads

$$
\delta_{k}^{(0)} \mathcal{L}_{n} \approx 0,
$$

where $\delta_{k}^{(0)}$ is the free gauge transformation of the field $\phi_{k}$ (see eq. (1.1)).

The latter can be simplified by contracting the tensor for the gauge parameter in eq. (1.1) with auxiliary vector variables $a^{\mu}$ as well,

$$
\epsilon^{(s-1)}(x, a)=\frac{1}{(s-1) !} \epsilon_{\mu_{1} \ldots \mu_{s-1}}(x) a^{\mu_{1}} \cdots a^{\mu_{s-1}} .
$$

Again, we drop the spin index, $\epsilon_{k}=\epsilon^{\left(s_{k}-1\right)}$, and the linearised gauge transformation of the $k$-th field $\phi_{k}$ in eq. (1.1) reads

$$
\delta_{k}^{(0)} \phi_{k}\left(x_{k}, a_{k}\right)=a_{k} \cdot P_{k} \epsilon_{k}\left(x_{k}, a_{k}\right), \quad \text { (no sum). }
$$

Note that this gauge transformation must be consistent with eqs. (2.3). We therefore impose the Fierz equations also for the gauge parameter.

All in all, we can now impose the restrictions for the vertex generating operators $\mathcal{V}$ from gauge invariance, eq. (2.12):

$$
\delta_{k}^{(0)} \mathcal{L}_{n}=\left.\mathcal{V} a_{k} \cdot P_{k}\left(\epsilon_{k}\left(x_{k}, a_{k}\right) \prod_{1 \leq i \leq n}^{i \neq k} \phi_{i}\left(x_{i}, a_{i}\right)\right)\right|_{\substack{x_{i}=x \\ a_{i}=0}} \approx 0
$$

Since all the auxiliary vector variables $a_{i}$ are set to zero in the end, it immediately follows that $\mathcal{L}_{n}$ is gauge invariant if and only if the corresponding vertex generating operator $\mathcal{V} \in \mathcal{R}$ (via eq. (2.4)) satisfies

$$
\text { for all } k \in\{1, \ldots, n\}: \quad\left[\mathcal{V}, a_{k} \cdot P_{k}\right]=: D_{k} \mathcal{V} \in \mathcal{I}_{S}+\mathcal{I}_{D}
$$


Here, we defined the operators $D_{k}$ of gauge variations. These act as linear first-order differential operators on the vertex $\mathcal{V}$ :

$$
D_{k}=\sum_{j=1}^{n}\left(y_{j k} \frac{\partial}{\partial z_{k j}}+s_{k j} \frac{\partial}{\partial y_{k j}}\right) .
$$

\section{The case $2 n-1 \leq d$}

We start with the case of sufficiently high spacetime dimensions where the classification of vertices is the simplest because there are no Schouten identities and we only have to take into account total derivatives, hence, $\mathcal{I}=\mathcal{I}_{D}$.

\subsection{Gauge invariants}

To derive the $n$-th order independent vertices we first recall the constraints on the vertex generating operators $y_{i j}, z_{i j}, s_{i j}$ in eqs. (2.6) and (2.7) and count the independent variables:

$$
\begin{aligned}
y_{i i} \approx 0, & \sum_{j=1}^{n} y_{i j} \approx 0, & & n(n-2) \text { variables } y_{i j}, \\
z_{i j}=z_{j i}, & z_{i i} \approx 0, & & \frac{n(n-1)}{2} \text { variables } z_{i j}, \\
s_{i j}=s_{j i}, & s_{i i} \approx 0, & \sum_{j=1}^{n} s_{i j} \approx 0, & \frac{n(n-3)}{2} \text { variables } s_{i j} .
\end{aligned}
$$

The vertex depends altogether on $2 n(n-2)$ variables, and is subject to $n$ linear differential equations that stem from eqs. (2.14) and (2.15). ${ }^{8}$ If these differential equations are linearly independent, the solution should depend on $2 n(n-2)-n=n(2 n-5)$ variables.

For cubic vertices, $n=3$, this would give three invariants, while we know that the solution depends on four invariants $y_{12}, y_{23}, y_{31}$ and $G=y_{12} z_{23}+y_{23} z_{31}+y_{31} z_{12}$. The reason is that the three equations are not linearly independent in that case: $y_{12} D_{1}+$ $y_{23} D_{2}+y_{31} D_{3} \approx 0$. Due to this relation, we have, e.g., the Yang-Mills cubic vertex $V_{3}^{Y M}=G$ and the Einstein-Hilbert cubic vertex $V_{3}^{E H}=G^{2}$.

On the other hand, one can easily see from eq. (2.15) that the operators $D_{k}$ are linearly independent for $n \geq 4$. Hence, the general form of the vertices should depend on $n(2 n-5)$ invariants composed of $s_{i j}, y_{i j}, z_{i j}$.

At this point, we introduce gauge invariant operators, which are more suitable as the building blocks of $n$-th order vertices. These are given through the following variables:

$$
\begin{array}{rlrl}
s_{i j} & =s_{j i} & & \frac{n(n-3)}{2} \text { variables }, \\
c_{i j} & =y_{i j} y_{j i}-s_{i j} z_{i j}=c_{j i}, & & \frac{n(n-1)}{2} \text { variables }, \\
c_{i, j k} & =y_{i j} s_{i k}-y_{i k} s_{i j}=-c_{i, k j}, & \frac{n(n-2)(n-3)}{2} \text { variables } .
\end{array}
$$

\footnotetext{
${ }^{8}$ Notice that the operators $D_{k}$ are consistent with these constraints (3.1), which means that $D_{k}$ acting on a constraint will lead to a constraint. Therefore we can leave the operators $D_{k}$ in the general form stated in eq. (2.15) and do not need to express them in terms of a set of independent variables.
} 
It is easy to show that these expressions are gauge invariant:

$$
D_{k} s_{i j}=0, \quad D_{k} c_{i j}=0, \quad D_{k} c_{i, j l}=0 .
$$

Counting the number of the variables $s_{i j}$ and $c_{i j}$ is straightforward. In order to count the number of $c_{i, j k}$ variables, we count separately the number of choices for $i$ and the number of choices for the antisymmetric pair $j k$ for a given $i$ and multiply them. Naively, we choose $i$ in $n$ possible ways, and the antisymmetric pair $j k$ takes values in $\{i+1, \ldots, i-2(\bmod \mathrm{n})\}$, therefore takes $\frac{(n-2)(n-3)}{2}$ values, hence the number of $c_{i, j k}$ 's given above. These variables $c_{i, j k}$ are not linearly independent though, satisfying the following relations:

$$
3 c_{i,[j k} s_{i \mid l]} \equiv c_{i, j k} s_{i l}+c_{i, k l} s_{i j}+c_{i, l j} s_{i k}=0 .
$$

These naively are $\frac{n(n-2)(n-3)(n-4)}{6}$ many, given by multiplying the $n$ possible choices of $i$ and $\frac{(n-2)(n-3)(n-4)}{6}$ choices of the antisymmetric triple $j k l$. But again, this counting is redundant, due to linear relations between equations, involving different choices of $j k l$. These relations are also given by adding another $s_{i m}$ and antisymmetrising the four indices $j k l m$. This chain of reducibility can be resummed to get all linearly independent variables of $c_{i, j k}$. This is done by finding the number of possible values of $j k$ antisymmetrised pairs that correspond to the independent variables, by summing up with changing signs the numbers of components of antisymmetric tensors of $g l(n-2)$, starting from rank two: ${ }^{9}$

$$
\sum_{i=2}^{n-2}(-1)^{i}\left(\begin{array}{c}
n-2 \\
i
\end{array}\right)=n-3 .
$$

This means that the number of independent variables $c_{i, j k}$ is $n(n-3)$. We see that the variables $c_{i, j k}$ are redundant and we choose the following set of independent variables:

$$
Y_{i}^{j}:=c_{i, i+j i+1}
$$

where now $j=2, \ldots, n-2$, taking $n-3$ possible values (indices are always meant modulo $n)$. Thus, the number of variables $Y_{i}^{j}$ is altogether $n(n-3)$. It is elementary to show that any other variable $c_{i, j k}$ can be expressed through $Y_{i}^{j}$ using eq. (3.6):

$$
c_{i, j k}=\frac{c_{i, j i+1} s_{i k}-c_{i, k i+1} s_{i j}}{s_{i i+1}}=\frac{Y_{i}^{j-i} s_{i k}-Y_{i}^{k-i} s_{i j}}{s_{i i+1}} .
$$

Therefore altogether we have:

$$
\frac{n(n-3)}{2}+\frac{n(n-1)}{2}+n(n-3)=n(2 n-5) \text { invariants. }
$$

Given that the number of independent invariants $s_{i j}, c_{i j}, Y_{i}^{j}$ is the same as the number of variables that should constitute the building blocks of $n$-th order independent vertices, it is already tempting to conclude that the most general solution is an arbitrary function of

\footnotetext{
${ }^{9}$ Remind, that the index $j$ takes $n-2$ independent values in $y_{i j}$ (and therefore in $c_{i, j k}$ ) due to (3.1a).
} 
these variables. We will show this now, by allowing for dividing by Mandelstam variables and making the replacements

$$
z_{i j}=\frac{1}{s_{i j}}\left(y_{i j} y_{j i}-c_{i j}\right)
$$

and, consecutively,

$$
y_{i i+j}=\frac{1}{s_{i i+1}}\left(y_{i i+1} s_{i i+j}-Y_{i}^{j}\right), \quad j=2, \ldots, n-2 \bmod n,
$$

expressing the vertex operator in terms of the variables $s_{i j}, c_{i j}, Y_{i}^{j}$ and $y_{i i+1}$. Correspondingly, the gauge variation in terms of these variables is generated by the operators

$$
D_{k}=s_{k k+1} \frac{\partial}{\partial y_{k k+1}},
$$

which turn into a single derivative. Therefore, the new gauge invariance equations for the vertex operator give:

$$
D_{k} \mathcal{V}\left(s_{i j}, c_{i j}, Y_{i}^{j}, y_{i i+1}\right)=s_{k k+1} \frac{\partial}{\partial y_{k k+1}} \mathcal{V}\left(s_{i j}, c_{i j}, Y_{i}^{j}, y_{i i+1}\right) \approx 0
$$

If we go to a set of independent variables, we can conclude that the $y_{i i+1}$-derivative is equal to zero, and the vertex can be solely written in terms of the gauge invariant combinations $s_{i j}, c_{i j}, Y_{i}^{j}$. A gauge invariant local vertex generating operator $\mathcal{V}$ in high enough dimension $(d \geq 2 n-1)$ is then in one-to-one correspondence to a polynomial in $s_{i j}, c_{i j}, Y_{i}^{j}$, allowing inverse powers of Mandelstam variables in such a way that $\mathcal{V}$ becomes polynomial in the variables $s_{i j}, y_{i j}$ and $z_{i j}$, when re-expressing the combinations $c_{i j}$ and $Y_{i}^{j}$.

\subsection{Building blocks of vertices}

We have just shown that any gauge-invariant vertex $\mathcal{V}$ of order $n$ for $d \geq 2 n-1$ can be rewritten as a function of the invariants $c_{i j}, Y_{i}^{j}$ and $s_{i j}$. This function is polynomial in $c_{i j}$ and $Y_{i}^{j}$, but can contain inverse powers of the Mandelstam variables $s_{i j}$.

In this subsection we address the question: 'what is the most general form of this function if we assume that the vertex is local?' First of all it is clear that any polynomial of $c_{i j}, Y_{i}^{j}$ and $s_{i j}$ defines a local and gauge-invariant vertex. Now let us analyse the case that the vertex contains a single pole in one $s_{i j}$ when written in terms of the invariants:

$$
\mathcal{V}=\frac{1}{s_{i j}} \mathcal{P}\left(c_{i j}, Y_{i}^{j}, s_{k l}\right)
$$

Here, we assume that the polynomial $\mathcal{P}$ does not explicitly depend on this specific $s_{i j}$. For $\mathcal{V}$ to be local, the inverse of $s_{i j}$ has to be compensated by a term proportional to $s_{i j}$ that arises when the invariants are rewritten in terms of $s_{i j}, y_{i j}, z_{i j}$. One can show that in this case, $\mathcal{V}$ is a linear combination of

$$
b_{i j k \ell}=\frac{1}{s_{i j}}\left(c_{i j} s_{i k} s_{j \ell}-c_{i, j k} c_{j, i \ell}\right) \quad \text { and } \quad \frac{1}{s_{i j}}\left(s_{i k} c_{i, j \ell}-s_{i \ell} c_{i, j k}\right)
$$


multiplied by polynomials in $c_{i j}, Y_{i}^{j}$ and the Mandelstam variables. ${ }^{10}$ The second expression is simply equal to $c_{i, k \ell}$ (see eq. (3.6)), so it is again a polynomial in $s_{k l}$ and $c$ variables. The first one can be rewritten as

$$
b_{i j k \ell}=\operatorname{det}\left(\begin{array}{lll}
s_{i j} & s_{i k} & y_{j i} \\
s_{\ell j} & s_{\ell k} & y_{j \ell} \\
y_{i j} & y_{i k} & z_{i j}
\end{array}\right)+s_{k \ell} c_{i j}
$$

Hence, up to a shift by a polynomial in Mandelstam and $c$ variables, the building block $b_{i j k \ell}$ can be written as a determinant of a $3 \times 3$-submatrix of the matrix $\mathcal{B}$ (see eq. (2.8)). This nicely fits with the observation that also the $c$ invariants are just minors of $\mathcal{B}$,

$$
c_{i j}=-\operatorname{det}\left(\begin{array}{ll}
s_{i j} & y_{j i} \\
y_{i j} & z_{i j}
\end{array}\right) \quad, \quad c_{i, j k}=\operatorname{det}\left(\begin{array}{cc}
s_{i k} & s_{i j} \\
y_{i k} & y_{i j}
\end{array}\right) .
$$

Notice that these minors as well as the $(3 \times 3)$-example above have the property that each $(n+i)$-th row (column) of the second block is accompanied by the corresponding $(i$-th) row (column) of the first block. This ensures gauge invariance because the $i$-th gauge variation transforms the $(n+i)$-th row (column) into the $i$-th row (column) leading to a vanishing determinant. Translating such a building block to the fields, the resulting expression is a pure curvature term: a tensor index of a field $i$ occurs in an antisymmetric combination with an index of a derivative acting on the field.

Of course all such minors can be written as polynomials in the $c$ invariants with negative powers of Mandelstam variables allowed. This can be explicitly seen when in the determinant we add to the $(j+n)$-th column the $j$-th column multiplied by $-\frac{y_{j j+1}}{s_{j j+1}}$, and similarly we add to the $(i+n)$-th row the $i$-th row multiplied by $-\frac{y_{i i+1}}{s_{i i+1}}$. Then one arrives at

$$
\left.\operatorname{det}\left(\begin{array}{ll}
\left(s_{i j}\right) & \left(y_{j i}\right) \\
\left(y_{i j}\right) & \left(z_{i j}\right)
\end{array}\right)=\operatorname{det}\left(\begin{array}{cc}
\left(s_{i j}\right) & \left(\frac{1}{s_{j j+1}} c_{j, i j+1}\right) \\
\left(\frac{1}{s_{i i+1}} c_{i, j i+1}\right) & \left(\frac{1}{s_{i j} s_{i i+1} s_{j j+1}}\left(c_{j, j+1 i} c_{i, i+1 j}-s_{i i+1} s_{j j+1} c_{i j}\right)\right.
\end{array}\right)\right) .
$$

Here, the labels $i$ and $j$ only run through the values that correspond to the rows and columns present in the minor that we are considering.

There is one additional possibility due to the linear dependencies in $\mathcal{B}$ : we can take the determinant of the $(2 n-1) \times(2 n-1)$ submatrix that is obtained by deleting, e.g., the first row and column. This is still gauge invariant because the gauge transformation with respect to the variables of the first field transforms the first row of the second block into a linear combination of the $n-1$ rows of the first block, and the determinant still vanishes. Expressed in terms of fields, such a building block corresponds to a term of the form

$$
\delta_{\nu_{2} \cdots \nu_{2 n}}^{\left[\mu_{2} \cdots \mu_{2 n}\right]} \phi_{\mu_{n+1}}^{(1) \nu_{n+1}} \partial_{\mu_{2}} \partial^{\nu_{2}} \phi_{\mu_{n+2}}^{(2) \nu_{n+2}} \cdots \partial_{\mu_{n}} \partial^{\nu_{n}} \phi_{\mu_{2 n}}^{(n) \nu_{2 n}},
$$

which is gauge invariant up to total derivatives. This Lovelock-type vertex can be generalised in a way, where one computes the determinant of the minor of $\mathcal{B}$ containing $n-1$

\footnotetext{
${ }^{10}$ Note that $c_{i, j k}$ can be expressed as a polynomial in $Y$ 's and Mandelstam variables via eq. (3.9).
} 
rows and columns from the first block and arbitrary number $m$ of rows and columns from the second block, but these do not introduce new building blocks. ${ }^{11}$

Note that also the Mandelstam variables $s_{i j}$ are $1 \times 1$-minors. It is tempting to speculate that all gauge invariant local vertices $\mathcal{V}$ can be written as polynomials in the types of minors of $\mathcal{B}$ mentioned above. If this speculation is correct, then for a spin configuration $s_{1} \geq s_{2} \geq \cdots \geq s_{n}(n \geq 4)$ the lowest number of derivatives in a parity-even local vertex is $s_{1}+s_{2}+\cdots+s_{n}-2\left\lfloor\frac{s_{n}}{2}\right\rfloor$, and is achieved only for $\bar{s}_{i}=s_{i}-2\left\lfloor\frac{s_{n}}{2}\right\rfloor(i=1, \ldots, n)$ satisfying polygon inequalities: $\bar{s}_{1} \leq \bar{s}_{2}+\cdots+\bar{s}_{n}$. In fact, taking into account the results of section 6 , we can make a stronger statement for the special case $d=2 n-1$ : if the polygon inequalities between quantities $\tilde{s}_{i}=s_{i}-s_{n}(i=1, \ldots, n-1)$ are satisfied $\left(\tilde{s}_{1} \leq \tilde{s}_{2}+\cdots+\tilde{s}_{n-1}\right)$, the lowest number of derivatives in a vertex is $N\left(s_{i}\right)=s_{1}+\cdots+s_{n-1}$, where the corresponding vertex is parity-odd for odd $s_{n}$. When these polygon inequalities are not satisfied, the number of derivatives in the local vertex will be higher than $N\left(s_{i}\right)$.

\section{Lower dimension: dealing with Schouten identities}

In the previous section we have discussed the gauge-invariant vertices when we do not have to consider Schouten identities. When we go to lower dimensions, the ideal of relations is enlarged from $\mathcal{I}_{D}$ to $\mathcal{I}_{D}+\mathcal{I}_{S}$. Gauge-invariant vertex generating operators for large dimensions still define gauge-invariant operators in lower dimensions, but a priori, enlarging the ideal could have two effects: first, inequivalent vertices become equivalent, and second, new vertices arise that are gauge-invariant only up to the now larger set of equivalence relations. We will show in the following that the latter possibility does not lead to new equivalence classes of vertices for $n \geq 4$, but that for all gauge-invariant vertex generating operators there are equivalent operators ${ }^{12}$ which are gauge-invariant already without the use of Schouten identities. While this holds for quartic and all higher vertices in arbitrary dimensions, for cubic vertices $(n=3)$ dimension-dependent vertices appear precisely in dimension $d=3$ (studied in $[8,9]$ ). ${ }^{13}$

To show this, we start with a vertex generating operator $\mathcal{V}$ as a polynomial in $s_{i j}, y_{i j}, z_{i j}$ that in $d$ dimensions is gauge invariant,

$$
D_{k} \mathcal{V} \in \mathcal{I}_{D}+\mathcal{I}_{S}
$$

In $\mathcal{V}$ we now express the variables $z_{i j}$ and $y_{i j}$ in terms of $c_{i j}, Y_{i}^{j}$ and $y_{i i+1}$,

$$
\mathcal{V}=\mathcal{P}_{\mathcal{V}}\left(c_{i j}, Y_{i}^{j}, y_{i i+1}\right)
$$

\footnotetext{
${ }^{11}$ By adding total derivatives they can be transformed to an expression of the type (3.19) where the $n-1$ rows (columns) of the first block contain the $m$ rows (columns) corresponding to those of the second block.

${ }^{12}$ As long as we can divide by Mandelstam variables.

${ }^{13}$ We would like to make a side remark here on the cubic vertices of Fronsdal fields in $d=2$, discussed in the appendix $\mathrm{B}$ of [9]. It can be shown, that the vertex $(s, s, 0)$ is also trivial (due to the Schouten identity $y_{i} y_{i+1} z_{i-1} \approx 0$ in the notations of [9]) for $s \geq 2$. The only non-trivial parity-even vertices remaining are thus $\mathcal{V}_{(1,1,0)}=y_{1} y_{2}, \mathcal{V}_{(s, s, 1)}=y_{3} z_{3}^{s}$ and the current coupling $\mathcal{V}_{(s, 0,0)}=y_{1}^{s}$ that has the same form as in arbitrary dimensions (the latter was forgotten in [9]). Note, that the on-shell triviality is not a reason to exclude the vertices as long as they cannot be removed via a local field redefinition, even if we assume that Fierz equations (which are stronger than free Fronsdal equations) can be removed by a field redefinition. This subtlety is discussed in detail in [48].
} 
where $\mathcal{P}_{\mathcal{V}}$ is a polynomial in the given variables. We suppressed the dependence on Mandelstam variables, which can also occur with negative powers. In these variables, the gauge variation $D_{k}$ is written as a derivative with respect to $y_{k k+1}$ as in eq. (3.13), so we have

$$
D_{k} \mathcal{V}=s_{k k+1} \frac{\partial}{\partial y_{k k+1}} \mathcal{P}_{\mathcal{V}}\left(c_{i j}, Y_{i}^{j}, y_{i i+1}\right) \in \mathcal{I}_{D}+\mathcal{I}_{S}
$$

When we expand $\mathcal{P}_{\mathcal{V}}$ in powers of $y_{12}$,

$$
\mathcal{P}_{\mathcal{V}}\left(c_{i j}, Y_{i}^{j}, y_{i i+1}\right)=\sum_{k=0}^{K} q_{k}\left(c_{i j}, Y_{i}^{j}, y_{23}, \ldots, y_{n 1}\right)\left(y_{12}\right)^{k},
$$

we apply $\left(D_{1}\right)^{K}$ to the expression and obtain

$$
K !\left(s_{12}\right)^{K} q_{K} \in \mathcal{I}_{D}+\mathcal{I}_{S} .
$$

When we allow ourselves to divide by Mandelstam variables, we conclude that

$$
q_{K} \in \frac{1}{\left(s_{12}\right)^{K}}\left(\mathcal{I}_{D}+\mathcal{I}_{S}\right) .
$$

Similar relations can be found for all other terms in the expansion in $y_{12}$ and also in the other variables $y_{i i+1}$. Hence, we find that

$$
\mathcal{V}-\left.\mathcal{P}_{\mathcal{V}}\left(c_{i j}, Y_{i}^{j}, y_{i i+1}\right)\right|_{y_{i i+1}=0} \in \frac{1}{\Delta}\left(\mathcal{I}_{D}+\mathcal{I}_{S}\right)
$$

where $\Delta$ is a product of powers of Mandelstam variables. Therefore, $\mathcal{V}$ is equivalent to an operator depending only on $c_{i j}$ and $Y_{i}^{j}$ which already defines a gauge invariant vertex operator without the need of Schouten identities.

We conclude that in all dimensions, vertex generating operators can be expressed in terms of the operators identified for large dimensions. The main task for lower dimensions is therefore to work out explicitly the equivalences between such operators that are induced by Schouten identities. Here, the case of low dimensions, $d<n$, is special because many Schouten identities arise that reduce the independent equivalence classes considerably. This will be discussed in detail in the subsequent section. The identifications in the intermediate case will be stated in the discussion in section 7 .

In the remainder of this section we give a heuristic geometric argument why generically one does not expect new vertices to appear when we lower the dimension. In the sense of algebraic geometry, the ideal $\mathcal{I}=\mathcal{I}_{S}+\mathcal{I}_{D}$ defines a variety $V(\mathcal{I})$ as the zero-set of the polynomials contained in $\mathcal{I}$. If $\mathcal{I}$ was a prime ideal, we could think of the $\operatorname{ring} \mathcal{R} / \mathcal{I}$ as the ring of polynomial functions on this variety. The gauge variations $D_{k}$ define $n$ vector fields on this variety, and we are looking for functions on $V(\mathcal{I})$ that are constant along the vector fields. When we enlarge the ideal to $\mathcal{I}^{\prime} \supset \mathcal{I}$ by going from higher to lower dimensions where new Schouten identities occur, we concentrate on a subvariety $V\left(\mathcal{I}^{\prime}\right)$ of $V(\mathcal{I})$. Generically, if the vector fields do not degenerate on this subvariety, functions that are constant along $D_{k}$ on $V\left(\mathcal{I}^{\prime}\right)$ can be lifted to constant functions on $V(\mathcal{I})$. 
The above argument only gives a very rough picture, because apart from the possible degeneration of the vector fields, there are two subtleties: first, as it was said, the argument only applies to prime ideals, but the ideals that occur are usually not prime; secondly, there could be constant polynomials on $V\left(\mathcal{I}^{\prime}\right)$ whose lifts to $V(\mathcal{I})$ are not polynomial. Therefore, this picture can only be seen as a heuristic explanation why generically we do not expect new gauge invariant vertices to appear when we lower the dimension.

\section{The case $n>d$}

In this section, we find general restrictions for gauge invariant $n$-point vertices with $n>d$. Our result is a simple characterisation of equivalence classes $[\mathcal{V}] \in \mathcal{R} / \mathcal{I}$ for vertex generating operators. The results are summarised in section 5.4.

As discussed in section 2.2, we have the full set of Schouten identities at hand in order to find a simple representative $\mathcal{V}$ for a given vertex. This has the advantage that a lot of simplifications are possible. On the other hand, the structure of the set of Schouten identities is complicated, and the number of linearly independent Schouten identities in the polynomial ring, $\frac{1}{2}\left(\begin{array}{c}2 n-1 \\ d+1\end{array}\right)\left(\left(\begin{array}{c}2 n-1 \\ d+1\end{array}\right)+1\right)$ for $n \geq 4$, is large and rapidly growing with $n$. This problem was solved in [1] for $d=3$ by observing that many Schouten identities become dependent when multiplied with an appropriate product $\Delta$ of Mandelstam variables. By multiplying a given vertex $\mathcal{V}$ with $\Delta$, the remaining independent Schouten identities can be used to deduce strong constraints for the vertex $\mathcal{V}$ itself. Essentially, one can treat the Mandelstam variables in the manipulations like numbers and also divide by them. This concept can be also employed in higher dimensions.

Formally, to be able to divide by certain combinations of Mandelstam variables, we introduce the ring of fractions, $M^{-1} \mathcal{R}$. Here, $M$ is a multiplicatively closed set containing all (finite) products of non-zero minors of the submatrix $\mathcal{S}$ of $\mathcal{B}$ (see eq. (2.8)): these are the expressions we want to divide by. More explicitly, let $\operatorname{Mi}(\mathcal{S})$ be the set of non-zero minors of $\mathcal{S},{ }^{14}$ and let $M=\operatorname{Mon}[\operatorname{Mi}(\mathcal{S})]$ be the set of monomials in these minors. Then, the ring of fractions consists of formal quotients,

$$
M^{-1} \mathcal{R}=\left\{\frac{r}{\Delta} \mid \Delta \in M, r \in \mathcal{R}\right\}
$$

with the obvious rules for addition and multiplication. As also $1 \in M$, we can identify $\mathcal{R}$ via $r \mapsto \frac{r}{1}$ as subring of $M^{-1} \mathcal{R}$. The ideal $\mathcal{I}=\mathcal{I}_{S}+\mathcal{I}_{D} \subset \mathcal{R}$ can then be seen as a subset of $M^{-1} \mathcal{R}$ which generates an ideal $\mathcal{I}_{M}$ in $M^{-1} \mathcal{R}$. Using the embedding of $\mathcal{R}$ into $M^{-1} \mathcal{R}$, we have an induced map of the quotient rings,

$$
i_{M}: \frac{\mathcal{R}}{\mathcal{I}} \rightarrow \frac{M^{-1} \mathcal{R}}{\mathcal{I}_{M}}
$$

\footnotetext{
${ }^{14}$ First, non-zero minors of order one are just the Mandelstam variables $s_{i j}$ with $i \neq j$. Secondly, all minors of order $2,3, \ldots, d$ are generically non-zero - even when the equivalence relations in eq. (2.7) are applied. Finally, all minors of order greater than $d$ do vanish due to Schouten identities. $\operatorname{Hence}, \operatorname{Mi}(\mathcal{S})$ consists of all $(2 \times 2),(3 \times 3), \ldots(d \times d)$ subdeterminants of $\mathcal{S}$ as well as the Mandelstam variables $s_{i j}$ with $i \neq j$.
} 
As we will argue below, this map is injective, and therefore we can characterise equivalence classes of vertices uniquely by equivalence classes in the ring of fractions. The crucial observation is now that in $M^{-1} \mathcal{R}$ many of the generators of the ideal become dependent, so that $\mathcal{I}_{M}$ has a simple set of generators.

This section is structured as follows. In section 5.1 we find a simple set of generators for $\mathcal{I}_{M}$, which enables us to find a convenient representative of $[\mathcal{V}]$ in the quotient of the ring of fractions in section 5.2. We then impose gauge invariance in section 5.3, which leads to strong restrictions on the vertex $\mathcal{V}$. In $d=3$, these restrictions completely rule out independent vertices (as reported in [1]), in higher dimensions the restrictions are less strict, and we discuss them in section 5.4. In order to make the structure of this paper better accessible, we collect some proofs in section 5.5.

Before we proceed, we want to show that $i_{M}$ is indeed injective. If $i_{M}([\mathcal{V}])=[0]$, this means that $\mathcal{V} \in \mathcal{R} \cap \mathcal{I}_{M}$. Then, there is some $\Delta \in M$ such that $\Delta \mathcal{V} \in \mathcal{I}$. If $\Delta \mathcal{V}$ defines a trivial vertex, then also $\mathcal{V}$ corresponds to a trivial vertex, which can be seen in Fourier space, where the operators $s_{i j}$ are numbers. In particular, the polynomial $\Delta$ is non-zero on the subvariety defined by $k_{i}^{2}=0$ and $\sum k_{i}=0$. Now, if $\Delta \mathcal{V}$ defines a trivial vertex, then

$$
\left.\Delta \mathcal{V} \prod_{i} \widehat{\phi}_{i}\left(k_{i}, a_{i}\right)\right|_{a_{i}=0}
$$

vanishes on this subvariety. The factor $\Delta$ is non-vanishing almost everywhere. Hence, since $\mathcal{V}$ only depends polynomially on $k_{i}^{\mu}, \mathcal{V}$ applied on the fields $\widehat{\phi}_{i}$ must vanish. So we conclude that $\mathcal{V} \approx 0$, hence $[\mathcal{V}]=[0]$.

\subsection{A minimal generating set of Schouten identities}

In this section, we find a simple set of generators for the ideal $\mathcal{I}_{M}$ in two steps. First, any Schouten identity multiplied with a certain $\Delta \in M=\operatorname{Mon}[\operatorname{Mi}(\mathcal{S})]$ is an element in the ideal generated by the equivalence relations in eq. (2.7) and all Schouten identities up to level $2^{15}$ (recall the notion of level introduced in the paragraph before eq. (2.10)). In other words,

$$
\text { there exists } \Delta \in \operatorname{Mon}[\operatorname{Mi}(\mathcal{S})] \text { such that } \Delta \cdot \mathcal{I}_{S} \subset \sum_{k=0}^{2} I(k)+\mathcal{I}_{D} \text {. }
$$

We show this in section 5.5.1. This observation implies that in the ring of fractions where we are allowed to divide by $\Delta$, we need far less generators for the Schouten identities.

In order to perform the second step, we introduce some more notations: first,

$$
N_{i j}=\left(\begin{array}{ccc}
s_{i j} & \cdots & s_{i j+d-1} \\
\vdots & \ddots & \vdots \\
s_{i+d-1 j} & \cdots & s_{i+d-1 j+d-1}
\end{array}\right)
$$

is a $d \times d$ submatrix of $\mathcal{S}$, hence, $\operatorname{det} N_{i j} \in \operatorname{Mi}(\mathcal{S})$ and $N_{i j}$ has full rank. Secondly, let $B_{1}(i, j)$ with $i, j=1, \ldots, n$ be the following $(d+1) \times(d+1)$ submatrix of $\mathcal{B}$ : it contains

\footnotetext{
${ }^{15}$ This proof relies on the fact that $n>d$.
} 
the rows and columns $i, i+1, \ldots, i+d-1$ (modulo $n$ ) as well as another row $j$ and the column $i+n$. Hence,

$$
\operatorname{det} B_{1}(i, j)=\operatorname{det}\left(\begin{array}{ccc} 
& & 0 \\
& & y_{i i+1} \\
N_{i i} & \vdots \\
& & y_{i i+d-1} \\
s_{j i} \cdots & s_{j i+d-1} & y_{i j}
\end{array}\right) \in I(1) \cdot{ }^{16}
$$

Finally, let $B_{2}(i, j)$ with $i, j=1, \ldots, n$ be the $(d+1) \times(d+1)$ submatrix of $\mathcal{B}$ containing the rows $i, i+1, \ldots, i+d-1$ (modulo $n$ ) and $i+n$, as well as the columns $j, j+1, \ldots, j+d-1$ (modulo $n$ ) and $j+n$. Hence,

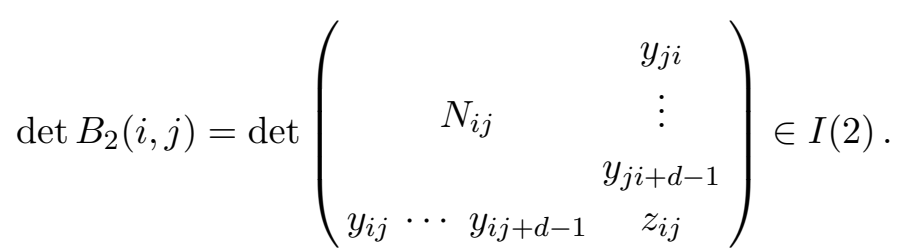

With these notations, we show in section 5.5.2 that there exists $\Delta \in M=\operatorname{Mon}[\operatorname{Mi}(\mathcal{S})]$ such that

$$
\Delta \cdot\left(\mathcal{I}_{S}+\mathcal{I}_{D}\right) \subset I(0)+\left\langle\sum_{k=1}^{n} s_{i k}, \operatorname{det} B_{1}(i, j), \operatorname{det} B_{2}(i, j) \mid i, j=1, \ldots, n\right\rangle .
$$

Denote the family of generators of $I(0)$ by $\left(\operatorname{det} B_{0}(A)\right)$, where $A$ labels the different equivalence relations. Then, we can conclude that $\mathcal{I}_{M}$ is generated as

$$
\mathcal{I}_{M}=\left\langle\sum_{k=1}^{n} s_{i k},\left(\operatorname{det} B_{0}(A)\right), \operatorname{det} B_{1}(i, j), \operatorname{det} B_{2}(i, j) \mid i, j=1, \ldots, n\right\rangle .
$$

\subsection{The choice of representative}

Now, let us investigate the relevant ideal $\mathcal{I}_{M}$ in order to choose a convenient representative for $\mathcal{V}$ in its equivalence class $[\mathcal{V}] \in M^{-1} \mathcal{R} / \mathcal{I}_{M}$.

We start by considering the Schouten identities $\operatorname{det} B_{2}(i, j) \in I(2)$, with $i \neq j$. Using a Laplace expansion along the last column, they read

$$
0 \approx \operatorname{det} B_{2}(i, j)=z_{i j} \operatorname{det} N_{i j}+\text { terms that do not contain any } z_{k l} .
$$

Since $\operatorname{det} N_{i j} \in \operatorname{Mi}(\mathcal{S})$, we can divide by it in $M^{-1} \mathcal{R}$, and express $z_{i j}$ by an expression independent of any $z_{k l}$. Hence, we may choose the representative of $[\mathcal{V}]$ to be independent of $z_{i j}$. In the same way, the Schouten identities $\operatorname{det} B_{1}(i, j) \in I(1)$ take the form

$$
0 \approx \operatorname{det} B_{1}(i, j)=y_{i j} \operatorname{det} N_{i i}+p\left(s_{i j}, y_{i i+1}, \ldots, y_{i i+d-1}\right) .
$$

\footnotetext{
${ }^{16}$ Note that this is true for all $j=1, \ldots, n$. If for example $j=i$, then $\operatorname{det} B_{1}(i, j)=0 \in I(1)$.
} 
Here, the polynomial $p$ only depends on $y_{i i+1}, \ldots, y_{i i+d-1},{ }^{17}$ and the Mandelstam variables. Using these Schouten identities, we can replace all of the operators $y_{i j}$ in $\mathcal{V}$ except for $y_{i i+1}, \ldots, y_{i i+d-1}$.

Finally, we perform a change of variables in $\mathcal{V}$. Similarly to eq. (3.8) we introduce the combinations

$$
Y_{i}^{j}=s_{i i+1} y_{i i+j}-s_{i i+j} y_{i i+1} \quad \text { for } j=2, \ldots, d-1,
$$

and replace all $y_{i i+2}, \ldots, y_{i i+d-1}$ in terms of these variables and $y_{i i+1}$. This can be done, because $s_{i i+1} \in M$ and we can divide by it in $M^{-1} \mathcal{R}$. We arrive at

$$
\mathcal{V} \approx \mathcal{P}_{\mathcal{V}}\left(y_{i i+1}, Y_{i}^{j}, s_{i j}\right),
$$

where $\mathcal{P}_{\mathcal{V}}$ is a polynomial in $y_{i i+1}, Y_{i}^{j}$ and the Mandelstam variables (with coefficients that can contain inverse powers of elements in $\operatorname{Mi}(\mathcal{S})$ ). More explicitly, we can see $[\mathcal{V}]$ as an element in the quotient

$$
[\mathcal{V}] \in \frac{M^{-1} \mathbb{R}\left[y_{i i+1}, Y_{i}^{j}, s_{i j}\right]}{\left\langle\left(\operatorname{det} B_{0}(A)\right), \sum_{j=1}^{n} s_{i j}, \operatorname{det} B_{2}(i, i) \mid i=1, \ldots, n\right\rangle} .
$$

There are several reasons to introduce the $Y_{i}^{j}$ variables. First, they are the gauge invariant combinations of the $y_{i j}$ variables - we have discussed this already in section 3 and it will become important in section 5.3. Secondly, the remaining level-2 Schouten identities $\operatorname{det} B_{2}(i, i)$ can be written solely in terms of the $Y_{i}^{j}$ 's and the Mandelstam variables, and they do not depend explicitly on $y_{i i+1}$. We show this in the remainder of this section: for this purpose, consider

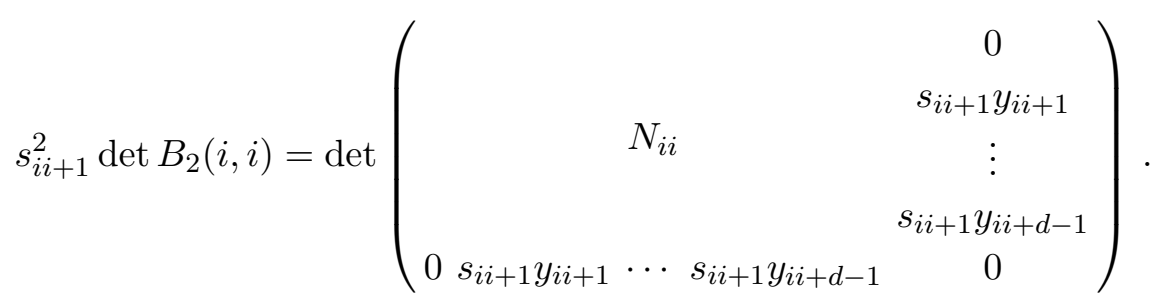

The determinant of the matrix does not change when $y_{i i+1}$ times the first row is subtracted from the last one and $y_{i i+1}$ times the first column is subtracted from the last one. Hence, using the definition of $Y_{i}^{j}$ in eq. (5.10), we find

$$
\begin{aligned}
s_{i i+1}^{2} \operatorname{det} B_{2}(i, i) & =\operatorname{det}\left(\begin{array}{rr}
0 \\
& 0 \\
N_{i i} & Y_{i}^{2} \\
\vdots \\
& Y_{i}^{d-1} \\
& 0
\end{array}\right) \\
& =-\sum_{j, k=2}^{d-1} Y_{i}^{j}\left(\operatorname{adj} N_{i i}\right)_{j k} Y_{i}^{k}=: q_{2}^{i}\left(Y_{i}^{j}, s_{j k}\right) .
\end{aligned}
$$

\footnotetext{
${ }^{17}$ The indices are considered modulo $n$.
} 
Here, we used a Laplace expansion along the last row and column, and adj $N_{i i}$ denotes the adjugate matrix of $N_{i i}$. The resulting polynomials $q_{2}^{i}$ are quadratic in the $Y_{i}^{j}$ variables with coefficients that still depend on the Mandelstam variables. However, the $q_{2}^{i}$ 's are independent of $y_{i i+1}$. We comment on their structure in section 5.4. All in all, we can replace the generators det $B_{2}(i, i)$ by $q_{2}^{i}$ because we are allowed to divide by Mandelstam variables. Hence, we have the following result:

$$
[\mathcal{V}] \in \frac{M^{-1} \mathbb{R}\left[y_{i i+1}, Y_{i}^{j}, s_{i j}\right]}{\left\langle\left(\operatorname{det} B_{0}(A)\right), \sum_{j=1}^{n} s_{i j}, q_{2}^{i} \mid i=1, \ldots, n\right\rangle} .
$$

\subsection{General restrictions from gauge invariance}

With the results of the previous sections, we now show that the polynomial $\mathcal{P} \mathcal{V}$ introduced in eq. (5.11) can be chosen to be independent of $y_{i i+1}$ if the operator $\mathcal{V}$ corresponds to a gauge invariant Lagrangian $\mathcal{L}_{n}$. From now on, we will always consider $\mathcal{V}$ as an element in the bigger ring of fractions.

Starting from eq. (2.14) and using that the operators $a_{k} \cdot P_{k}$ commute with all Mandelstam variables, we find that a gauge invariant vertex $\mathcal{L}_{n}$ requires

$$
\text { for all } k \in\{1, \ldots n\}: \quad\left[\mathcal{V}, a_{k} \cdot P_{k}\right] \in \mathcal{I}_{M} \text {, }
$$

where $\mathcal{L}_{n}$ and $\mathcal{V}$ are related via eq. (2.4). Now, since the ideal $\mathcal{I}_{M}$ is gauge invariant, $\left[\mathcal{I}_{M}, a_{k} \cdot P_{k}\right] \subset \mathcal{I}_{M}$, we deduce that the polynomial in eq. (5.11) satisfies

$$
\left[\mathcal{P}_{\mathcal{V}}, a_{k} \cdot P_{k}\right] \in\left\langle\left(\operatorname{det} B_{0}(A)\right), \sum_{j=1}^{n} s_{i j}, q_{2}^{i}\left(Y_{i}^{j}\right) \mid i=1, \ldots, n\right\rangle
$$

With

$$
\left[y_{i i+1}, a_{k} \cdot P_{k}\right]=\delta_{i k} s_{i i+1} \quad \Rightarrow \quad\left[Y_{i}^{j}, a_{k} \cdot P_{k}\right]=\delta_{i k}\left(s_{i i+j} s_{i i+1}-s_{i i+1} s_{i i+j}\right)=0,
$$

it follows immediately that

$$
\text { for all } k=1, \ldots, n: \quad s_{k k+1} \partial_{y_{k k+1}} \mathcal{P}_{\mathcal{V}} \in\left\langle\left(\operatorname{det} B_{0}(A)\right), \sum_{j=1}^{n} s_{i j}, q_{2}^{i}\left(Y_{i}^{j}\right) \mid i=1, \ldots, n\right\rangle \text {. }
$$

The generators of the ideal on the r.h.s. do not depend on $y_{i i+1}$. We conclude that $\mathcal{P}_{\mathcal{V}}$ can be chosen to be independent of $y_{i i+1}$. More explicitly,

$$
\mathcal{V} \approx \mathcal{P}_{\mathcal{V}}\left(Y_{i}^{j}, s_{i j}\right), \quad[\mathcal{V}] \in \frac{M^{-1} \mathbb{R}\left[Y_{i}^{k}, s_{i j}\right]}{\left\langle\left(\operatorname{det} B_{0}(A)\right), \sum_{j=1}^{n} s_{i j}, q_{2}^{i}\left(Y_{i}^{j}\right) \mid i=1, \ldots, n\right\rangle} .
$$

\subsection{Restrictions for $\mathcal{V}$}

Let us summarise our results. Eq. (5.13) states that each gauge invariant vertex $\mathcal{V}$ is equivalent to a vertex $\mathcal{P}_{\mathcal{V}}$ that does only depend on Mandelstam variables and $Y_{i}^{j}$. In 
particular, translating back to the vertex in terms of $P_{i}^{\mu}$ and $A_{i}^{\mu}$ operators, we have the following relation:

$$
\iota_{d}\left(Y_{i}^{j}\right)=2 P_{i \mu} A_{i \nu} P_{[i+1}^{\mu} P_{i+j]}^{\nu}=2 P_{i[\mu} A_{|i| \nu]} P_{i+1}^{\mu} P_{i+j}^{\nu} .
$$

Now, in the vertex generated by $\mathcal{P} \mathcal{V}$, an index of the $i$ th field is only generated by $A_{i}^{\mu}$ via a corresponding $Y_{i}^{j}$. Hence, the $i$ th field enters the Lagrangian via a curvature term (each index of the field is antisymmetrised with an index of a partial derivative acting on it). We deduce that $\mathcal{P}_{V}$ generates a Lagrangian that can be written solely in terms of curvature terms.

The drawback of this analysis is that we do not control locality on the way to this result. $\mathcal{P}_{\mathcal{V}}$ might not have a local form, since it can have inverse powers of Mandelstam variables. We can only say that for each gauge invariant vertex (generated by $\mathcal{V}$ ), there is a $\Delta \in M$ such that $\Delta \mathcal{V}$ can be written only in terms of curvatures.

Much stricter conditions can be found in three dimensions [1]. In that case, there is only one $Y_{i}^{j}$ and the corresponding Schouten identity is $q_{2}^{i}=-s_{i i+1}^{2}\left(Y_{i}^{2}\right)^{2}$. Hence, $\operatorname{det} B_{2}(i, i)=\left(Y_{i}^{2}\right)^{2} \approx 0$ and $\mathcal{P}_{\mathcal{V}}$ is only linear in $Y_{i}$. One can then deduce that $\mathcal{V}$ itself is at most linear in each of the operators $A_{i}^{\mu}$, which means that the corresponding vertex $\mathcal{L}_{n}$ contains no higher-spin fields at all. Indeed, in $d=3$ there are simply no on-shell non-trivial curvature terms for higher-spin fields.

Our analysis also applies to the case $d=2$. Here, no $Y_{i}^{j}$ remain, and therefore there are no independent vertices for $n \geq 4$ involving massless Fronsdal fields of spin $s \geq 1$.

\subsection{Proofs}

\subsubsection{Proof of eq. (5.4)}

Let $\operatorname{det} M=0$ be a Schouten identity that stems from a $(d+1) \times(d+1)$-submatrix $M$ of $\mathcal{B}$ such that $\operatorname{det} M \notin \mathcal{I}_{D}$. Let $r(s)$ be the number of level-0 rows (columns) of $M$. Furthermore, let $\bar{r}(\bar{s})$ be the number of level-1 rows (columns) of $M$. Hence, $r+\bar{r}=$ $s+\bar{s}=d+1$. Without loss of generality, we assume $r \geq s .{ }^{18}$ Furthermore, let $\bar{s} \geq 2$, hence, the level of the Schouten identity $\operatorname{det} M=0$ is $\bar{r}+\bar{s} \geq 2$. In particular, equality holds if and only if $\bar{s}=2$ and $\bar{r}=0$.

With the submatrix $M$ given, we construct a $(d+2) \times(d+2)$-submatrix $\widetilde{M}$ of $\mathcal{B}$ as follows:

- Removing $(2 n-d-2)$ rows and columns from $\mathcal{B}$ results in $\widetilde{M}$.

- There is a level-0 row (which we call Row) and a level-0 column (called $C o l$ ) in $\widetilde{M}$, such that removing Row and $C o l$ in $\widetilde{M}$ yields $M$. Hence, $\widetilde{M}$ contains $(r+1)$ level- 0 rows and $(s+1)$ level-0 columns.

- The construction of $\widetilde{M}$ might not be unique, but is always possible. This can be seen as follows: first, there is at least one level-0 row of $\mathcal{B}$ that is not part of $M$ (otherwise,

\footnotetext{
${ }^{18}$ If $r<s$, we choose $M^{T}$ instead of $M$, which yields the same Schouten identity $\operatorname{det} M^{T}=\operatorname{det} M . M^{T}$ is a submatrix of $\mathcal{B}$ as well because $\mathcal{B}$ is symmetric.
} 

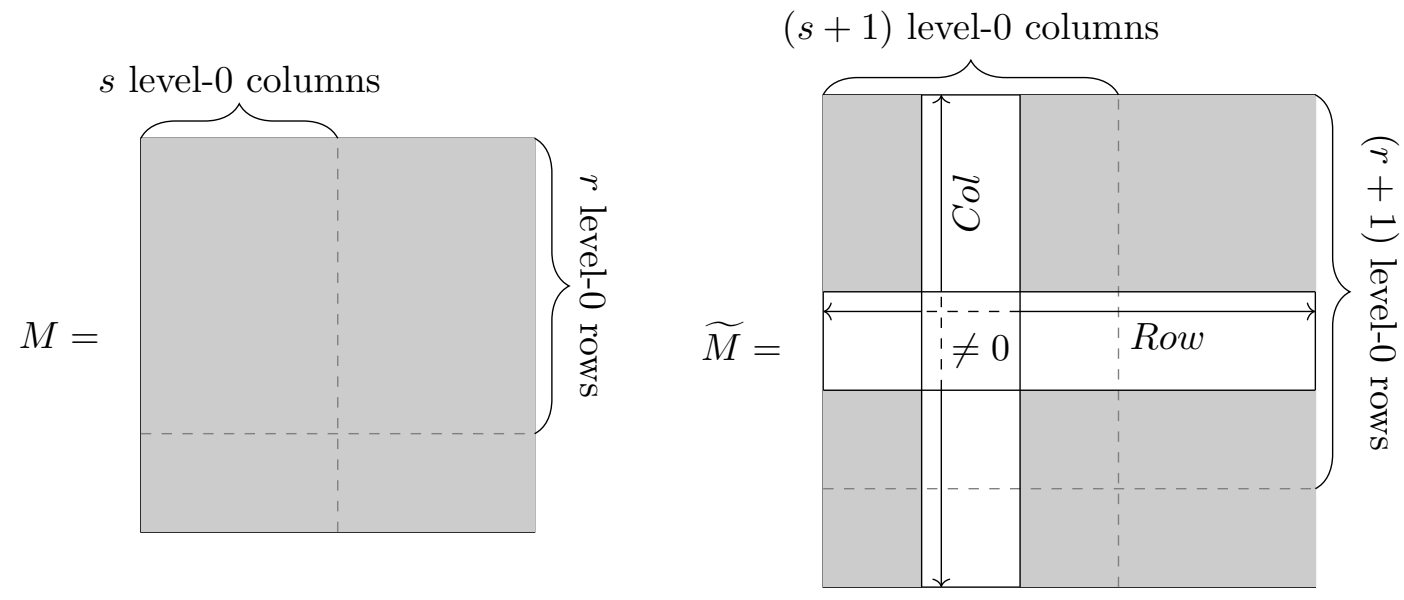

Figure 1. Visualisation of the matrices $M$ and $\widetilde{M}$.

$M$ would contain all level-0 rows of $\mathcal{B}$ which means that $\operatorname{det} M \in \mathcal{I}_{D}$ which contradicts our assumption). Furthermore, there are at least two level-0 columns of $\mathcal{B}$ that are not part of $M$, because $\bar{s} \geq 2$ and hence, $s \leq d-1 .{ }^{19}$ In particular, we can always choose $\widetilde{M}$ such that the intersection of Row and $C o l$ contains a non-zero Mandelstam variable.

The construction of the matrix $\widetilde{M}$ is visualised in figure 1 .

For $\widetilde{M}$, Cramers rule states that

$$
\mathbb{I}_{(d+2) \times(d+2)} \operatorname{det} \widetilde{M}-\widetilde{M} \cdot C^{T}=0,
$$

where $C=\left(c_{i j}\right)$ denotes the cofactor matrix of $\widetilde{M}=\left(\widetilde{m}_{i j}\right)$. In particular, $c_{i j}$ is (up to a factor of \pm 1$)$ equal to the determinant of the $(d+1) \times(d+1)$-submatrix obtained by deleting the $i$-th row and the $j$-th column from $\widetilde{M}$. In other words, $c_{i j}$ is a $(d+1) \times(d+1)$-minor of $\mathcal{B}$, hence $c_{i j} \in \mathcal{I}_{S}$. In the following, we consider only part of eq. (5.14):

$$
\delta_{j i} \operatorname{det} \widetilde{M}-\sum_{k=1}^{s+1} \widetilde{m}_{j k} c_{i k}-\sum_{k=s+2}^{d+2} \widetilde{m}_{j k} c_{i k}=0 \quad i=1, \ldots, s+1, j \in J .
$$

Here, $J$ is a (non-unique) subset of $s+1$ level-0 rows that contains Row. In other words,

$$
J \subset\{1, \ldots, r+1\}, \quad|J|=s+1, \quad \text { Row } \in J .
$$

Performing a Laplace expansion of $\operatorname{det} \widetilde{M}$ along the last column of $\widetilde{M}$ (which is of level 1 because of $\bar{s} \geq 2$ ), we deduce that $\operatorname{det} \widetilde{M}$ is a linear combination of Schouten identities of level $\bar{r}+\bar{s}-1$ and $\bar{r}+\bar{s}-2$. Hence,

$$
\operatorname{det} \widetilde{M} \in I(\bar{r}+\bar{s}-1)+I(\bar{r}+\bar{s}-2) .
$$

\footnotetext{
${ }^{19} \mathcal{B}$ has more than $d$ level-0 columns, since $n>d$.
} 
Furthermore, in the third term of eq. (5.15), the Schouten identities $c_{i k}$ with $k>s+1$ are of level $(\bar{r}+\bar{s}-1)$. We therefore conclude that the middle term is an element in the following ideal:

$$
\text { for all } i=1, \ldots, s+1, j \in J:\left(\sum_{k=1}^{s+1} \widetilde{m}_{j k} c_{i k}\right) \in I(\bar{r}+\bar{s}-1)+I(\bar{r}+\bar{s}-2) \text {. }
$$

Now, denote by $N=\left(\widetilde{m}_{j k}\right)$ (with $j \in J$ and $\left.k \in\{1, \ldots, s+1\}\right)$ the $(s+1) \times(s+1)$ submatrix of $\widetilde{M}$ that occurs in eq. (5.16). It is also a submatrix of $\mathcal{S}$ because it only consists of level-0 rows and columns. Since $s+1 \leq d$, we deduce that $\operatorname{det} N \in \operatorname{Mi}(\mathcal{S}) .{ }^{20} \mathrm{In}$ particular, $\operatorname{det} N \neq 0$ and by inverting $N$ in eq. (5.16) using Cramers rule, we find

$$
\text { for all } j \in J, k \in\{1, \ldots, s+1\}: \quad \operatorname{det} N \cdot c_{j k} \in I(\bar{r}+\bar{s}-1)+I(\bar{r}+\bar{s}-2) .
$$

Finally, setting $j=R o w$ and $k=C o l$, we have $c_{j k}=\operatorname{det} M-$ which corresponds to the Schouten identity of level $(\bar{r}+\bar{s})$ we started with. It follows directly that for all $\operatorname{det} M \in I(\bar{r}+\bar{s})$, either $\operatorname{det} M \in \mathcal{I}_{D}$ or

$$
\exists \operatorname{det} N \in \operatorname{Mi}(\mathcal{S}): \quad \operatorname{det} N \cdot \operatorname{det} M \in I(\bar{r}+\bar{s}-1)+I(\bar{r}+\bar{s}-2) .
$$

In other words,

$$
\exists \Delta \in \operatorname{Mon}[\operatorname{Mi}(\mathcal{S})]: \quad \Delta \cdot I(\bar{r}+\bar{s}) \subset I(\bar{r}+\bar{s}-1)+I(\bar{r}+\bar{s}-2)+\mathcal{I}_{D}
$$

and a recursion over $\bar{r}$ and $\bar{s}$ proves the general statement in eq. (5.4).

\subsubsection{Proof of eq. (5.7)}

We prove eq. (5.7) in three steps. It directly follows from eq. (5.4), as well as eqs. (5.18), (5.22 and 5.26).

Part 1: first of all, we show that

$$
\exists \Delta \in \operatorname{Mon}[\operatorname{Mi}(\mathcal{S})]: \quad \Delta I(2) \subset I(0)+I(1)+\left\langle\operatorname{det} B_{2}(i, j) \mid i, j=1, \ldots, n\right\rangle .
$$

For this purpose, consider an arbitrary level-2 Schouten identity $\operatorname{det} M \approx 0$, with $M$ being a $(d+1) \times(d+1)$-submatrix of $\mathcal{B}$. As explained in the previous section, we may assume that $M$ has either one $(\bar{r}=1, \bar{s}=1)$ or two level-1 columns $(\bar{r}=0, \bar{s}=2)$. In the latter case, the proof of the previous section goes through and eq. (5.17) is satisfied. Hence, we only need to consider the other case $(\bar{r}=\bar{s}=1)$. In particular, we show that if $M$ contains the level-1 row $i+n$ and the level- 1 column $j+n$ of $\mathcal{B}$, then:

$$
\exists \Delta \in \operatorname{Mon}[\operatorname{Mi}(\mathcal{S})]: \quad \Delta \operatorname{det} M \in I(1)+\left\langle\operatorname{det} B_{2}(i, j)\right\rangle,
$$

which implies eq. (5.18).

We prove eq. (5.19) by induction. For the following discussion, we fix $i$ and $j$. Let $I_{2}(K, L)$ be the ideal generated by all level-2 Schouten identities $\operatorname{det} M \approx 0$, such that the $(d+1) \times(d+1)$-submatrix $M$ of $\mathcal{B}$ has the following properties:

\footnotetext{
${ }^{20}$ In the case that $s=0, N$ is just a Mandelstam variable. But within the construction of $\widetilde{M}$, we chose Row and $C o l$ such that its intersection (which is $N$ in that case) is non-zero. Therefore, $\operatorname{det} N=N \neq 0$.
} 
i) $M$ contains the level-1 row $i+n$ and the level- 1 column $j+n$ of $\mathcal{B}$.

ii) $K$ rows ( $L$ columns) of $M$ stem from the rows $i, \ldots, i+d-1$ (columns $j, \ldots, j+d-1$ ) (modulo $n$ ) of $\mathcal{B}$.

Hence, $I_{2}(d, d)=\left\langle\operatorname{det} B_{2}(i, j)\right\rangle$ and eq. (5.19) is a recursive consequence of the following two propositions:

$$
\begin{array}{cll}
\text { if } K<d, & \exists \Delta \in \operatorname{Mon}[\operatorname{Mi}(\mathcal{S})]: & \Delta \cdot I_{2}(K, L) \subset I(1)+I_{2}(K+1, L), \\
\text { if } L<d, & \exists \Delta \in \operatorname{Mon}[\operatorname{Mi}(\mathcal{S})]: & \Delta \cdot I_{2}(K, L) \subset I(1)+I_{2}(K, L+1) .
\end{array}
$$

Here, we present the proof of eq. (5.21). Eq. (5.20) can be shown in the same way, except that the roles of rows and columns are interchanged. We start with a $(d+1) \times(d+1)$ submatrix $M$ of $\mathcal{B}$, such that $\operatorname{det} M \in I_{2}(K, L)$ with $L<d$. For $M$ given, we construct a $(d+2) \times(d+2)$-matrix $\widetilde{M}$ as follows:

- Removing the first row from $\widetilde{M}$ yields a $(d+1) \times(d+2)$-submatrix $\widehat{M}$ of $\mathcal{B}$.

- There is a unique $k_{0} \in\{1, \ldots, d+1\}$, such that removing the $k_{0}$-th column from $\widehat{M}$ yields $M$. We construct $\widetilde{M}$ such that the $k_{0}$-th column stems from one of the columns $j, \ldots, j+d-1$ (modulo $n$ ) of $\mathcal{B}$, which is possible because $L<d$.

- Note that $\widehat{M}$ has $d+1$ level-0 columns. Hence, at least one of those cannot stem from one of the columns $j, \ldots, j+d-1$ (modulo $n$ ) of $\mathcal{B}$. Let us agree that at least the $l_{0}$-th column has this property. Obviously, $l_{0} \neq k_{0}$.

- The first two rows of $\widetilde{M}$ coincide, hence, $\operatorname{det} \widetilde{M}=0$.

Now, Cramers rule states that $\widetilde{M} C^{T}=0$, where $C=\left(c_{k l}\right)$ is the cofactor matrix of $\widetilde{M}=\left(\widetilde{m}_{k l}\right)$. The first column of this matrix equation reads

$$
\sum_{l=1}^{d+2} \widetilde{m}_{k l} c_{1 l}=0 .
$$

Note that up to a factor of $\pm 1, c_{1 l}$ is the determinant of the $(d+1) \times(d+1)$-matrix obtained by removing the first row and the $l$ th column from $\widetilde{M}$. Hence, $c_{1 l} \in I(2)$ for $l \leq d+1$ and $c_{1 d+2} \in I(1)$. In particular, $c_{1 k_{0}} \propto \operatorname{det} M \in I_{2}(K, L)$ and $c_{1 l_{0}} \propto \operatorname{det} M_{l_{0} \rightarrow k_{0}} \in I_{2}(K, L+1)$, where the matrix $M_{l_{0} \rightarrow k_{0}}$ differs from $M$ by only one column. Indeed, it contains the $k_{0}$-th column of $\widetilde{M}$ instead of the $l_{0}$-th. We deduce that

$$
\sum_{1 \leq l \leq d+1}^{l \neq l_{0}} \widetilde{m}_{k l} c_{1 l} \in I(1)+I_{2}(K, L+1) .
$$

Now, consider only the rows $2, \ldots, d+1$ of that relation. The matrix $N=\left(\widetilde{m}_{k l}\right)$ with $k \in\{2, \ldots, d+1\}$ and $l \in\{1, \ldots, d+1\} \backslash\left\{l_{0}\right\}$ is a $(d \times d)$-submatrix of $\mathcal{S}$ and can hence, be inverted using Cramers rule. We find that

$$
\operatorname{det} N \cdot c_{1 l} \in I(1)+I_{2}(K, L+1)
$$

and setting $l=k_{0}$ finally proves eq. (5.21). 
Part 2: in a second step, we show that

$$
\exists \Delta \in \operatorname{Mon}[\operatorname{Mi}(\mathcal{S})] \quad: \quad \Delta I(1) \subset I(0)+\left\langle\operatorname{det} B_{1}(i, j) \mid i, j=1, \ldots, n\right\rangle .
$$

The proof is similar to the previous one. Let $\operatorname{det} M \approx 0$ be an arbitrary level- 1 Schouten identity, where $M$ is a $(d+1) \times(d+1)$-submatrix of $\mathcal{B}$. As explained in the previous section, we may assume that $M$ has exactly one level-1 column $(\bar{r}=0, \bar{s}=1)$. In particular, we show that if $M$ contains the level-1 column $i+n$ of $\mathcal{B}$, then:

$$
\exists \Delta \in \operatorname{Mon}[\operatorname{Mi}(\mathcal{S})]: \quad \Delta \operatorname{det} M \in I(0)+\left\langle\operatorname{det} B_{1}(i, j) \mid j=1, \ldots, n\right\rangle,
$$

which implies eq. (5.22).

Again, we prove eq. (5.23) by induction for a fixed $i$. Let $I_{1}(K, L)$ be the ideal generated by all level-1 Schouten identities $\operatorname{det} M \approx 0$, such that the $(d+1) \times(d+1)$-submatrix $M$ of $\mathcal{B}$ has the following properties:

i) $M$ contains the level-1 column $i+n$ of $\mathcal{B}$.

ii) $K$ rows ( $L$ columns) of $M$ stem from the rows (columns) $i, \ldots, i+d-1$ (modulo $n$ ) of $\mathcal{B}$.

Hence, $I_{1}(d, d)=\left\langle\operatorname{det} B_{1}(i, j) \mid j=1, \ldots, n\right\rangle$ and eq. (5.23) is a recursive consequence of the following two propositions:

$$
\begin{array}{ccl}
\text { if } K<d, & \exists \Delta \in \operatorname{Mon}[\operatorname{Mi}(\mathcal{S})]: & \Delta \cdot I_{1}(K, L) \subset I(0)+I_{1}(K+1, L), \\
\text { if } L<d, & \exists \Delta \in \operatorname{Mon}[\operatorname{Mi}(\mathcal{S})]: & \Delta \cdot I_{1}(K, L) \subset I(0)+I_{1}(K, L+1) .
\end{array}
$$

Here, we give the proof of eq. (5.25), Eq. (5.24) follows analogously. For a given $(d+1) \times(d+1)$-submatrix $M$ of $\mathcal{B}$, such that $\operatorname{det} M \in I_{1}(K, L)$ with $L<d$, we construct a $(d+2) \times(d+2)$-matrix $\widetilde{M}$ as follows:

- Removing the first row from $\widetilde{M}$ yields a $(d+1) \times(d+2)$-submatrix $\widehat{M}$ of $\mathcal{B}$.

- There is a unique $k_{0} \in\{1, \ldots, d+1\}$, such that removing the $k_{0}$-th column from $\widehat{M}$ yields $M$. Again, we construct $\widetilde{M}$ such that the $k_{0}$-th column stems from one of the columns $i, \ldots, i+d-1$ (modulo $n$ ) of $\mathcal{B}$, which is possible because $L<d$.

- Note that $\widehat{M}$ has $d+1$ level-0 columns. Hence, at least one of those (say, the $l_{0}$-th) cannot stem from one of the columns $i, \ldots, i+d-1$ (modulo $n$ ) of $\mathcal{B}$. Again, $l_{0} \neq k_{0}$.

- The first two rows of $\widetilde{M}$ coincide, hence, $\operatorname{det} \widetilde{M}=0$.

Now, Cramers rule states that $\widetilde{M} C^{T}=0$, where $C=\left(c_{k l}\right)$ is the cofactor matrix of $\widetilde{M}=\left(\widetilde{m}_{k l}\right)$. Considering the first column of this matrix equation, we have

$$
\sum_{l=1}^{d+2} \widetilde{m}_{k l} c_{1 l}=0 .
$$


Up to a factor of $\pm 1, c_{1 l}$ is the determinant of the $(d+1) \times(d+1)$-matrix obtained by removing the first row and the $l$ th column from $\widetilde{M}$. Hence, $c_{1 l} \in I(1)$ for $l \leq d+1$ and $c_{1 d+2} \in I(0)$. In particular, $c_{1 k_{0}} \propto \operatorname{det} M \in I_{1}(K, L)$ and $c_{1 l_{0}} \propto \operatorname{det} M_{l_{0} \rightarrow k_{0}} \in I_{1}(K, L+1)$, where the matrix $M_{l_{0} \rightarrow k_{0}}$ differs from $M$ by only one column (it contains the $k_{0}$-th column of $\widetilde{M}$ instead of the $l_{0}$-th). We deduce that

$$
\sum_{1 \leq l \leq d+1}^{l \neq l_{0}} \widetilde{m}_{k l} c_{1 l} \in I(0)+I_{1}(K, L+1) .
$$

Again, we only consider the rows $2, \ldots, d+1$ of that relation. The matrix $N=\left(\widetilde{m}_{k l}\right)$ with $k \in\{2, \ldots, d+1\}$ and $l \in\{1, \ldots, d+1\} \backslash\left\{l_{0}\right\}$ is a $(d \times d)$-submatrix of $\mathcal{S}$ and can be inverted using Cramers rule. Finally,

$$
\operatorname{det} N \cdot c_{1 l} \in I(0)+I_{1}(K, L+1)
$$

and setting $l=k_{0}$ proves eq. (5.25).

Part 3: finally, we prove that for any $i \in\{1, \ldots, n\}$,

$$
\operatorname{det} N_{i i} \sum_{j=1}^{n} y_{i j} \in\left\langle\sum_{j=1}^{n} s_{i j}\right\rangle+\left\langle\operatorname{det} B_{1}(i, j)\right\rangle
$$

where $N_{i i} \in \operatorname{Mi}(\mathcal{S})$ is defined in eq. (5.5).

Fix $i \in\{1, \ldots, n\}$. Then, for any $j \in\{1, \ldots, n\}$, let $N_{i i}(k \rightarrow j)$ be the matrix $N_{i i}$, where the $(k+1)$ st row is replaced by $\left(s_{j i} s_{j i+1} \cdots s_{j i+d-1}\right)$. In particular,

$$
\sum_{j=1}^{n} \operatorname{det} N_{i i}(k \rightarrow j) \in\left\langle\sum_{j=1}^{n} s_{i j}\right\rangle
$$

because the determinant of $N_{i i}(k \rightarrow j)$ is linear (especially in the $(k+1)$ st row).

Now, a Laplace expansion of $\operatorname{det} B_{1}(i, j)$ (see eq. (5.6)) with respect to the last column results in

$$
\operatorname{det} B_{1}(i, j)=y_{i j} \operatorname{det} N_{i i}-\sum_{k=0}^{d-1} y_{i k+1} \operatorname{det} N_{i i}(k \rightarrow j),
$$

which holds for all $j \in\{1, \ldots, n\}$. In particular,

$$
\operatorname{det} N_{i i} \sum_{j=1}^{n} y_{i j}=\sum_{j=1}^{n} \operatorname{det} B_{1}(i, j)+\sum_{k=0}^{d-1} y_{i k+1} \sum_{j=1}^{n} \operatorname{det} N_{i i}(k \rightarrow j),
$$

which, taking eq. (5.27) into account, proves eq. (5.26).

\section{Parity-odd vertices}

So far, we only discussed parity-even vertices, i.e. terms in the Lagrangian which do not involve the epsilon tensor $\epsilon_{\mu_{1} \cdots \mu_{d}}$. However, the discussion of the previous sections can simply be generalised also for parity-odd vertices. 
First of all, the most general form of a parity-odd vertex is given by eq. (2.4) but with $\mathcal{V}$ replaced by

$$
\tilde{\mathcal{V}}=\sum_{I_{1} \cdots I_{d}} Q_{I_{1} \cdots I_{d}} \tilde{\mathcal{V}}^{I_{1} \cdots I_{d}}
$$

where $\tilde{\mathcal{V}}^{I_{1} \cdots I_{d}} \in \mathbb{R}\left[y_{i j},\left.z_{i j}\right|_{i \leq j},\left.s_{i j}\right|_{i \leq j}\right]$ contains the parity-even contractions $^{21}$ and

$$
Q_{I_{1} \cdots I_{d}}=\epsilon_{\mu_{1} \cdots \mu_{d}} b_{I_{1}}^{\mu_{1}} \cdots b_{I_{d}}^{\mu_{d}}
$$

is totally antisymmetric in its indices $\left(I_{k} \in\{1, \ldots, 2 n\}\right)$. The derivative operators $b_{I}$ were introduced in section 2.2, right before eq. (2.8). Note that for $i=1, \ldots, n$, we have $b_{i}=P_{i}$ and $b_{i+n}=A_{i}$. The structure of the gauge-invariant parity-odd vertices depends on the dimension:

- For $d \geq 2 n$, there are no parity-odd $n$-point vertex operators, because $Q_{I_{1} \cdots I_{d}}=0$ (the vector $b$ has only $2 n-1$ independent entries up to total derivatives).

- In the case $n>d$, we again make use of the fact that we consider $[\tilde{\mathcal{V}}]$ in the ring of fractions. The crucial point is that the general form of an elementary building block $Q_{I_{1} \cdots I_{d}}$ of parity-odd vertices can be highly simplified, when it is multiplied with the upper-left $d \times d$ submatrix of $\mathcal{S}$. Denote this matrix by $S_{d}$. Its determinant,

$$
\operatorname{det} S_{d}=\frac{1}{d !} \epsilon_{\mu_{1} \cdots \mu_{d}} \epsilon_{\nu_{1} \cdots \nu_{d}} b_{1}^{\mu_{1}} \cdots b_{d}^{\mu_{d}} b_{1}^{\nu_{1}} \cdots b_{d}^{\nu_{d}}
$$

is a non-zero minor of $\mathcal{S}$, hence, $\operatorname{det} S_{d} \in \operatorname{Mi}(\mathcal{S})$ and we conclude that

$$
\operatorname{det} S_{d} \cdot Q_{I_{1} \cdots I_{d}}=\left.\left(\mathcal{B}_{1 I_{1}} \cdots \mathcal{B}_{d I_{d}}\right)\right|_{\left[I_{1} \cdots I_{d}\right]} \cdot Q_{1 \cdots d} \text {. }
$$

In other words, for any parity-odd vertex in the Lagrangian given by the vertex generating operator $\tilde{\mathcal{V}}$ in eq. (6.1), we find

$$
\operatorname{det} S_{d} \cdot \tilde{\mathcal{V}}=Q_{1 \cdots d} \cdot \mathcal{V}
$$

where $\mathcal{V} \in \mathbb{R}\left[y_{i j},\left.z_{i j}\right|_{i \leq j},\left.s_{i j}\right|_{i \leq j}\right]$ as in the parity-even case.

Now, since we work in the ring of fractions, we can divide by $\operatorname{det} S_{d} \in \operatorname{Mi}(\mathcal{S})$. Furthermore, $Q_{1 \ldots d}$ is gauge invariant:

$$
\left[Q_{1 \cdots d}, a_{k} \cdot P_{k}\right]=0
$$

Hence, along the same lines as in section 5, we find that

$$
\tilde{\mathcal{V}} \approx Q_{1 \cdots d} \cdot \mathcal{P} \mathcal{V}\left(Y_{i}^{j}, s_{i j}\right)
$$

- The intermediate case, $n \leq d \leq 2 n-1$, can be tackled in a similar way. Let $S_{n-1}$ be the upper left $(n-1) \times(n-1)$ submatrix of $\mathcal{S}$. Up to a factor, its determinant is given by

$$
\operatorname{det} S_{n-1} \propto \epsilon_{\mu_{1} \cdots \mu_{d}} \epsilon_{\nu_{1} \cdots \nu_{n-1}} \mu_{n} \cdots \mu_{d} b_{1}^{\mu_{1}} \cdots b_{n-1}^{\mu_{n-1}} b_{1}^{\nu_{1}} \cdots b_{n-1}^{\nu_{n-1}} .
$$

\footnotetext{
${ }^{21}$ We discussed these in the previous sections where they were called $\mathcal{V}$.
} 
Multiplying it to the general vertex in eq. (6.1) yields

$$
\operatorname{det} S_{n-1} \tilde{\mathcal{V}} \propto \sum_{I_{1} \cdots I_{d}} \epsilon_{\nu_{1} \cdots \nu_{n-1}}{ }_{n} \cdots \mu_{d} b_{1}^{\mu_{1}} \cdots b_{n-1}^{\mu_{n-1}} b_{1}^{\nu_{1}} \cdots b_{n-1}^{\nu_{n-1}} b_{I_{1} \mu_{1}} \cdots b_{I_{d} \mu_{d}} \tilde{\mathcal{V}}^{\left[I_{1} \cdots I_{d}\right]} .
$$

We again work in the ring of fractions. Hence, we can divide by $\operatorname{det} S_{n-1}$, because it is a nonzero minor of $\mathcal{S}$. We finally find

$$
\tilde{\mathcal{V}} \approx \sum_{I_{n} \cdots I_{d}} Q_{1 \cdots n-1 I_{n} \cdots I_{d}} \hat{\mathcal{V}}^{I_{n} \cdots I_{d}}
$$

Here, we collect all direct parity even index contractions into one vertex generating operator $\hat{\mathcal{V}}^{I_{n} \cdots I_{d}}$, which is fully antisymmetric in its indices. Note that $I_{n}, \ldots, I_{d}>n$ because the $Q$-tensor is fully antisymmetric. ${ }^{22}$ In particular, with eq. (6.2), the $Q$ tensor reduces to a "square-root of a Horndeski-type operator",

$$
Q_{1 \cdots n-1 I_{n} \cdots I_{d}}=\epsilon_{\mu_{1} \cdots \mu_{d}} P_{1}^{\mu_{1}} \cdots P_{n-1}^{\mu_{n-1}} A_{I_{n}-n}^{\mu_{n}} \cdots A_{I_{d}-n}^{\mu_{d}} .
$$

It is trivially gauge invariant up to total derivatives:

$$
\left[Q_{1 \cdots n-1 I_{n} \cdots I_{d}}, a_{k} \cdot P_{k}\right] \approx 0 .
$$

Hence, as in the case $n>d$, we conclude that

$$
\tilde{\mathcal{V}} \approx \sum_{I_{n} \cdots I_{d}} Q_{1 \cdots n-1 I_{n} \cdots I_{d}} \cdot \mathcal{P}_{\mathcal{V}}^{I_{n} \cdots I_{d}}\left(Y_{i}^{j}, s_{i j}\right)
$$

where the polynomials $\mathcal{P}_{\mathcal{V}}^{I_{n} \cdots I_{d}}$ only depend on $Y_{i}^{j}$ and the Mandelstam variables. E.g. for $d=2 n-1$, there is only one term in the sum, namely

$$
\tilde{\mathcal{V}} \approx Q_{1 \cdots n-1 n+1 \cdots 2 n} \cdot \mathcal{P}_{\mathcal{V}}^{n+1 \cdots 2 n}\left(Y_{i}^{j}, s_{i j}\right) .
$$

Note that $Q_{1 \cdots n-1 n+1 \cdots 2 n}$ squares to the Lovelock operator (3.20). This covers also the case of $n=3$ and $d=5$, where $\mathcal{P}_{\mathcal{V}}$ is parity-even cubic vertex operator [6]. These covariant parity-odd $5 d$ vertices match the light-cone classification [5].

In all cases the vertices can be brought to a form in which they are gauge invariant without the use of Schouten identities. We conclude, that the situation with parity-odd vertices is analogous to the parity-even ones: Schouten identities do not give rise to new vertices except for the cubic ones in three dimensions, studied in [9].

\section{Discussion}

In this work, we complete the classification of independent vertices of arbitrary order $n \geq 3$ for massless bosonic fields with arbitrary spin in arbitrary spacetime dimensions $d \geq 2 .{ }^{23}$

\footnotetext{
${ }^{22}$ If one of those indices equals $n, Q_{1 \cdots n} I_{n+1} \cdots I_{d}$ vanishes equivalently because it equals a total derivative.

${ }^{23} \mathrm{We}$ concentrate on the traceless-transverse (TT) part of the vertices for classification, as discussed in the beginning of section 2 .
} 
We briefly summarise the results:

- For dimensions $d \geq 2 n-1$ there are no non-trivial Schouten identities. After reducing to the independent Mandelstam variables, we find that all gauge invariant operators can be expressed as polynomials in the gauge-invariant combinations $c_{i j}$ and $Y_{i}^{j}$,

$$
\mathcal{V} \in M_{1}^{-1} \mathbb{R}\left[s_{i j}, c_{i j}, Y_{i}^{j}\right]
$$

where $M_{1}$ is the set of all products of Mandelstam variables $s_{i j}(i \neq j)$. The invariant combinations $Y_{i}^{j}$ are labelled by $i=1, \ldots, n$ and $j=2, \ldots, n-2$.

- For dimensions $d<n$ we have the full set of Schouten identities at our disposal. All gauge invariant operators are already generated by the $Y_{i}^{j}$ 's, where $i=1, \ldots, n$ and $j=2, \ldots, d-1$. All remaining relations are generated by level-0 Schouten identities and specific quadratic expressions $q_{2}^{i}$ in the variables $Y_{i}^{j}$,

$$
[\mathcal{V}] \in \frac{M^{-1} \mathbb{R}\left[s_{i j}, Y_{i}^{j}\right]}{\left\langle\left(\operatorname{det} B_{0}(A)\right), q_{2}^{i}\right\rangle},
$$

where again we reduced to the independent Mandelstam variables.

- In the intermediate case $(2 n-1>d \geq n)$, we have Schouten identities, but because $d \geq n$ the non-trivial Schouten identities involve at least $(d-n)+2 \geq 2$ level-1 rows and columns. By an argument analogous to the one leading to eq. (5.4) one can show that in the ring of fractions all Schouten identities are generated by those that contain $n-1$ level-0 rows and columns and $(d-n)+2$ rows and columns of level-1. Let us denote them by $\operatorname{det} B_{2(d-n)+4}(A)$, where $A$ labels the possible choices of the level-1 rows and columns. These generators are all gauge-invariant (up to total derivatives), and hence we can express them in terms of the invariant combinations $c_{i j}$ and $Y_{i}^{j}$ as in section 3 . Then the gauge invariant vertices are classified by equivalence classes

$$
[\mathcal{V}] \in \frac{M^{-1} \mathbb{R}\left[s_{i j}, c_{i j}, Y_{i}^{j}\right]}{\left\langle\operatorname{det} B_{2(d-n)+4}(A)\right\rangle} .
$$

An interesting question is whether the higher order vertices can induce deformations of gauge transformations for the fields involved. Deformations arise when the gauge variation is non-trivial before imposing the equations of motion. Terms in the variation that contain the equations of motion have to be compensated by a non-trivial $\delta^{(n-2)}$ in eq. (1.3). We have found that in all dimensions, as long as we are allowed to divide by Mandelstam variables, the independent gauge-invariant vertices can be expressed in terms of the combinations $c_{i j}$ and $Y_{i}^{j}=c_{i, i+j i+1}$, but these - as defined in eq. (3.3) and eq. (3.4) - are manifestly gauge-invariant without need of the equations of motion. This strongly suggests that the vertex does not induce a deformation. Strictly speaking we can only conclude that $\Delta \mathcal{V}$ for an appropriate product $\Delta$ of Mandelstam variables does not induce any deformation. However, in Fourier space $\Delta$ is simply a (generically non-zero) number and should not 
change the general structure of deformations, hence we do not expect that $\mathcal{V}$ itself can induce a deformation. ${ }^{24}$

To recapitulate, as soon as we allow for dividing by Mandelstam variables (and hence, we loose manifest locality), the independent vertices of order $n \geq 4$ can be all written in terms of linearised curvatures of HS fields. Therefore they are manifestly gauge invariant with respect to linearised gauge transformations and do not introduce deformations for the latter. On the other hand, if such deformations of the gauge transformations, induced from cubic vertices, exist in the theory, then these vertices will be completed by further non-linear terms. This is similar to higher-curvature terms in Einstein Gravity, whose nonlinear structure is gauge invariant with respect to full diffeomorphisms, induced from the Einstein-Hilbert cubic vertex. Such non-linear completions may make use of a non-linear generalisation of de Wit-Freedman curvatures [86], which are not known in the metric-like formulation (see, however, [87]). In the frame formulation, these vertices would correspond to structures that make use of Weyl tensors and their descendants (zero form sector of the Vasiliev system). In light of our findings here, the three dimensional results of [1] can be interpreted as a particular case of the general dimensional results: all the independent vertices are given through linearised curvatures, which are on-shell trivial in $d=3$.

Even though the classification is done for Minkowski spaces, we expect the vertices found here to deform smoothly to $(A) d S$ spacetimes as it happens for cubic vertices. Indeed, the existence of $(A) d S$ extensions for linearised de Wit-Freedman curvatures for HS fields [88] allows to straightforwardly lift vertices given through curvatures to $(A) d S_{d}$. This is also true for the operators (3.20) and their parity-odd counterparts given in section 6 , where one can simply replace derivatives with $(A) d S_{d}$ covariant ones.

Our results should have a direct analogue for correlation functions of conserved tensors in $d-1$ dimensional conformal field theories, which can be classified with similar methods [89]. For $n=3$ there is a precise match between independent vertices and three-point functions [5-7, 39, 48, 49, 89-91]. It would be interesting to compare our findings for $n \geq 4$ with the group theoretic results of [92].

Next, we would like to note that there is another interpretation of eq. (2.12) which we solved here. One can think of eq. (2.12) as a Ward identity for an $n$-point amplitude computed in a theory of interacting HS fields. It is clear from our discussion, that the building blocks of the amplitudes are given through $c_{i j}, Y_{i}^{j}=c_{i, i+j i+1}$ and Mandelstam variables, including negative powers of the latter. They correspond to arbitrary tensor contractions of linearised curvatures [86] of HS gauge fields and their derivatives. These linear de Wit-Freedman curvatures (or their traceless part: the Weyl tensors) and their derivatives are the only on-shell non-zero gauge invariants with respect to the linearised gauge transformations. It is natural that the amplitudes for $n \geq 4$ should be given through gauge invariant quantities, as they are observable.

The amplitude interpretation might be less motivated in three dimensions since there are no propagating HS massless particles in three dimensions. As proved in [1], there

\footnotetext{
${ }^{24}$ This fits nicely with the observation obtained within the BRST formalism for spins up to $s=4$ that deformations of the gauge algebra can only arise from cubic vertices $[60,84,85]$ and observations about some quartic vertices [66, 67].
} 
are no candidate invariants for amplitudes with such fields either for $d=3$. There is one difference between amplitudes and vertices though - the latter are supposed to be local, while the former do not have to. Given that one can always multiply the candidate invariant vertices (amplitudes) by a non-vanishing function of Mandelstam variables, one can show that relaxing locality would not help to get non-zero amplitudes in $d=3$. There is an interesting conclusion to be made here: since the amplitude is a sum of exchanges ${ }^{25}$ and contact vertices, vanishing amplitudes imply that the exchanges and contact vertices should cancel each other. This is only possible if the non-local parts of the exchanges sum up to zero, which should be specific to three dimensions and is presumably due to the special structure of vertices and Schouten identities present only in three dimensions. We plan to study the Lagrangian formulation of metric-like non-linear HS theories with(out) matter in the near future to expose these special properties of HS gravities in $d=3$.

Note added. We learned from Euihun Joung and Massimo Taronna about their preprint with related results [95], which will appear on arxiv simultaneously.

\section{Acknowledgments}

The authors are grateful to Kostya Alkalaev, Xavier Bekaert, Dario Francia, Euihun Joung, Shailesh Lal, Ruben Manvelyan and Eugene Skvortsov for useful discussions on the subject of this work. KM is grateful to Max Planck Institute for Gravitational Physics (Albert Einstein Institute), where part of this work was done. The hospitality of the Erwin Schrödinger International Institute for Mathematics and Physics during the program on "Higher Spins and Holography" where this work was initiated is greatly appreciated. The work of KM was supported in part by Scuola Normale, by INFN (IS GSS-Pi) and by the MIUR-PRIN contract 2017CC72MK_003.

Open Access. This article is distributed under the terms of the Creative Commons Attribution License (CC-BY 4.0), which permits any use, distribution and reproduction in any medium, provided the original author(s) and source are credited.

\section{References}

[1] S. Fredenhagen, O. Krüger and K. Mkrtchyan, Vertex-constraints in 3D higher spin theories, Phys. Rev. Lett. 123 (2019) 131601 [arXiv:1905. 00093] [InSPIRE].

[2] A.K.H. Bengtsson, I. Bengtsson and N. Linden, Interacting higher spin gauge fields on the light front, Class. Quant. Grav. 4 (1987) 1333 [InSPIRE].

[3] R.R. Metsaev, Poincaré invariant dynamics of massless higher spins: fourth order analysis on mass shell, Mod. Phys. Lett. A 6 (1991) 359 [INSPIRE].

[4] R.R. Metsaev, $S$ matrix approach to massless higher spins theory. 2: the case of internal symmetry, Mod. Phys. Lett. A 6 (1991) 2411 [InSPIRE].

\footnotetext{
${ }^{25}$ The exchange is again a notion that is defined when there are particles to exchange, but this should not affect our argument, given that a propagator for massless HS fields can be formally defined in three dimensions. See, e.g., [93, 94]. We thank Shailesh Lal for a discussion about this point.
} 
[5] R.R. Metsaev, Cubic interaction vertices of massive and massless higher spin fields, Nucl. Phys. B 759 (2006) 147 [hep-th/0512342] [InSPIRE].

[6] R. Manvelyan, K. Mkrtchyan and W. Rühl, General trilinear interaction for arbitrary even higher spin gauge fields, Nucl. Phys. B 836 (2010) 204 [arXiv: 1003.2877] [INSPIRE].

[7] E. Conde, E. Joung and K. Mkrtchyan, Spinor-helicity three-point amplitudes from local cubic interactions, JHEP 08 (2016) 040 [arXiv: 1605.07402] [INSPIRE].

[8] K. Mkrtchyan, Cubic interactions of massless bosonic fields in three dimensions, Phys. Rev. Lett. 120 (2018) 221601 [arXiv:1712.10003] [INSPIRE].

[9] P. Kessel and K. Mkrtchyan, Cubic interactions of massless bosonic fields in three dimensions II: parity-odd and Chern-Simons vertices, Phys. Rev. D 97 (2018) 106021 [arXiv: 1803. 02737] [INSPIRE].

[10] A. Sagnotti and M. Taronna, String lessons for higher-spin interactions, Nucl. Phys. B 842 (2011) 299 [arXiv: 1006.5242] [INSPIRE].

[11] A. Fotopoulos and M. Tsulaia, On the tensionless limit of string theory, off-shell higher spin interaction vertices and BCFW Recursion relations, JHEP 11 (2010) 086 [arXiv: 1009.0727] [INSPIRE].

[12] R. Manvelyan, K. Mkrtchyan and W. Rühl, A generating function for the cubic interactions of higher spin fields, Phys. Lett. B 696 (2011) 410 [arXiv:1009.1054] [INSPIRE].

[13] K. Mkrtchyan, On generating functions of higher spin cubic interactions, Phys. Atom. Nucl. 75 (2012) 1264 [arXiv:1101.5643] [INSPIRE].

[14] A. Sagnotti, Notes on strings and higher spins, J. Phys. A 46 (2013) 214006 [arXiv: 1112.4285] [INSPIRE].

[15] R.R. Metsaev, BRST-BV approach to cubic interaction vertices for massive and massless higher-spin fields, Phys. Lett. B 720 (2013) 237 [arXiv:1205.3131] [INSPIRE].

[16] C. Fronsdal, Massless fields with integer spin, Phys. Rev. D 18 (1978) 3624 [InSPIRE].

[17] A. Campoleoni and D. Francia, Maxwell-like Lagrangians for higher spins, JHEP 03 (2013) 168 [arXiv: 1206.5877] [INSPIRE].

[18] M.A. Vasiliev, Consistent equation for interacting gauge fields of all spins in (3+1)-dimensions, Phys. Lett. B 243 (1990) 378 [InSPIRE].

[19] S.F. Prokushkin and M.A. Vasiliev, Higher spin gauge interactions for massive matter fields in 3D AdS space-time, Nucl. Phys. B 545 (1999) 385 [hep-th/9806236] [INSPIRE].

[20] M.A. Vasiliev, Nonlinear equations for symmetric massless higher spin fields in (A)dS(d), Phys. Lett. B 567 (2003) 139 [hep-th/0304049] [INSPIRE].

[21] X. Bekaert, S. Cnockaert, C. Iazeolla and M.A. Vasiliev, Nonlinear higher spin theories in various dimensions, hep-th/0503128 [INSPIRE].

[22] V.E. Didenko and E.D. Skvortsov, Elements of Vasiliev theory, arXiv:1401.2975 [INSPIRE].

[23] E. Joung and K. Mkrtchyan, Notes on higher-spin algebras: minimal representations and structure constants, JHEP 05 (2014) 103 [arXiv: 1401.7977] [INSPIRE].

[24] S.E. Konshtein and M.A. Vasiliev, Massless representations and admissibility condition for higher spin superalgebras, Nucl. Phys. B 312 (1989) 402 [INSPIRE]. 
[25] E. Joung, W. Li and M. Taronna, No-Go theorems for unitary and interacting partially massless spin-Two fields, Phys. Rev. Lett. 113 (2014) 091101 [arXiv:1406. 2335] [INSPIRE].

[26] E. Joung, K. Mkrtchyan and G. Poghosyan, Looking for partially-massless gravity, JHEP 07 (2019) 116 [arXiv: 1904.05915] [INSPIRE].

[27] S.E. Konstein and M.A. Vasiliev, Extended higher spin superalgebras and their massless representations, Nucl. Phys. B 331 (1990) 475 [InSPIRE].

[28] M.A. Vasiliev, Higher spin algebras and quantization on the sphere and hyperboloid, Int. J. Mod. Phys. A 6 (1991) 1115 [InSPIRE].

[29] E.S. Fradkin and M.A. Vasiliev, Cubic interaction in extended theories of massless higher spin fields, Nucl. Phys. B 291 (1987) 141 [inSPIRE].

[30] E.S. Fradkin and M.A. Vasiliev, On the gravitational interaction of massless higher spin fields, Phys. Lett. B 189 (1987) 89 [inSPIRE].

[31] E. Joung and M. Taronna, Cubic-interaction-induced deformations of higher-spin symmetries, JHEP 03 (2014) 103 [arXiv:1311.0242] [INSPIRE].

[32] C. Aragone and S. Deser, Consistency problems of hypergravity, Phys. Lett. 86B (1979) 161 [INSPIRE].

[33] S. Giombi, Higher spin - CFT Duality, in the proceedings of Theoretical Advanced Study Institute in Elementary Particle Physics: New Frontiers in Fields and Strings (TASI 2015), June 1-26, Boulder, U.S.A. (2015), arXiv:1607.02967 [INSPIRE].

[34] M.R. Gaberdiel and R. Gopakumar, Minimal model holography, J. Phys. A 46 (2013) 214002 [arXiv: 1207.6697] [INSPIRE].

[35] R. Manvelyan and K. Mkrtchyan, Conformal invariant interaction of a scalar field with the higher spin field in AdS(D), Mod. Phys. Lett. A 25 (2010) 1333 [arXiv:0903.0058] [INSPIRE].

[36] X. Bekaert, E. Joung and J. Mourad, On higher spin interactions with matter, JHEP 05 (2009) 126 [arXiv:0903.3338] [INSPIRE].

[37] K. Mkrtchyan, Linearized interactions of scalar and vector fields with the higher spin field in AdSD, Armenian J. Phys. 3 (2010) 98 [Phys. Part. Nucl. Lett. 8 (2011) 266].

[38] M.A. Vasiliev, Cubic vertices for symmetric higher-Spin gauge fields in (A)d $S_{d}$, Nucl. Phys. B 862 (2012) 341 [arXiv:1108.5921] [INSPIRE].

[39] E. Joung and M. Taronna, Cubic interactions of massless higher spins in (A)dS: metric-like approach, Nucl. Phys. B 861 (2012) 145 [arXiv:1110.5918] [INSPIRE].

[40] E. Joung, L. Lopez and M. Taronna, Solving the Noether procedure for cubic interactions of higher spins in (A)dS, J. Phys. A 46 (2013) 214020 [arXiv:1207.5520] [InSPIRE].

[41] R. Manvelyan, R. Mkrtchyan and W. Rühl, Radial reduction and cubic interaction for higher spins in (A)dS space, Nucl. Phys. B 872 (2013) 265 [arXiv:1210.7227] [INSPIRE].

[42] N. Boulanger, D. Ponomarev and E.D. Skvortsov, Non-Abelian cubic vertices for higher-spin fields in Anti-de Sitter space, JHEP 05 (2013) 008 [arXiv:1211.6979] [INSPIRE].

[43] X. Bekaert, J. Erdmenger, D. Ponomarev and C. Sleight, Towards holographic higher-spin interactions: four-point functions and higher-spin exchange, JHEP 03 (2015) 170 [arXiv: 1412.0016] [INSPIRE]. 
[44] X. Bekaert, J. Erdmenger, D. Ponomarev and C. Sleight, Quartic AdS interactions in higher-spin gravity from conformal field theory, JHEP 11 (2015) 149 [arXiv:1508.04292] [INSPIRE].

[45] C. Sleight and M. Taronna, Higher spin interactions from conformal field theory: the complete cubic couplings, Phys. Rev. Lett. 116 (2016) 181602 [arXiv:1603.00022] [INSPIRE].

[46] C. Sleight and M. Taronna, Higher-spin gauge theories and bulk locality, Phys. Rev. Lett. 121 (2018) 171604 [arXiv: 1704.07859] [INSPIRE].

[47] C. Sleight and M. Taronna, Feynman rules for higher-spin gauge fields on $A d S_{d+1}$, JHEP 01 (2018) 060 [arXiv:1708.08668] [INSPIRE].

[48] D. Francia, G.L. Monaco and K. Mkrtchyan, Cubic interactions of Maxwell-like higher spins, JHEP 04 (2017) 068 [arXiv: 1611.00292] [INSPIRE].

[49] C. Sleight and M. Taronna, Spinning Witten diagrams, JHEP 06 (2017) 100 [arXiv: 1702.08619] [INSPIRE].

[50] M. Karapetyan, R. Manvelyan and R. Poghossian, Cubic interaction for higher spins in $A d S_{d+1}$ space in the explicit covariant form, Nucl. Phys. B 950 (2020) 114876 [arXiv:1908.07901].

[51] M. Grigoriev and E.D. Skvortsov, Type-B Formal higher spin gravity, JHEP 05 (2018) 138 [arXiv: 1804.03196] [INSPIRE].

[52] A. Sharapov and E. Skvortsov, Formal higher spin gravities, Nucl. Phys. B 941 (2019) 838 [arXiv: 1901.01426] [INSPIRE].

[53] A.K.H. Bengtsson, I. Bengtsson and L. Brink, Cubic interaction terms for arbitrary spin, Nucl. Phys. B 227 (1983) 31 [InSPIRE].

[54] F.A. Berends, G.J.H. Burgers and H. van Dam, On the theoretical problems in constructing interactions involving higher spin massless particles, Nucl. Phys. B 260 (1985) 295 [INSPIRE].

[55] X. Bekaert, N. Boulanger, S. Cnockaert and S. Leclercq, On Killing tensors and cubic vertices in higher-spin gauge theories, Fortsch. Phys. 54 (2006) 282 [hep-th/0602092] [INSPIRE].

[56] N. Boulanger and S. Leclercq, Consistent couplings between spin-2 and spin-3 massless fields, JHEP 11 (2006) 034 [hep-th/0609221] [INSPIRE].

[57] D. Francia, J. Mourad and A. Sagnotti, Current exchanges and unconstrained higher spins, Nucl. Phys. B 773 (2007) 203 [hep-th/0701163] [INSPIRE].

[58] A. Fotopoulos and M. Tsulaia, Gauge invariant Lagrangians for free and interacting higher spin fields. A review of the BRST formulation, Int. J. Mod. Phys. A 24 (2009) 1 [arXiv:0805.1346] [INSPIRE].

[59] Yu.M. Zinoviev, On spin 3 interacting with gravity, Class. Quant. Grav. 26 (2009) 035022 [arXiv:0805.2226] [INSPIRE].

[60] N. Boulanger, S. Leclercq and P. Sundell, On the uniqueness of minimal coupling in higher-spin gauge theory, JHEP 08 (2008) 056 [arXiv:0805.2764] [INSPIRE].

[61] R. Manvelyan, K. Mkrtchyan and W. Rühl, Off-shell construction of some trilinear higher spin gauge field interactions, Nucl. Phys. B 826 (2010) 1 [arXiv:0903.0243] [INSPIRE]. 
[62] R. Manvelyan, K. Mkrtchyan and W. Rühl, Direct construction of a cubic selfinteraction for higher spin gauge fields, Nucl. Phys. B 844 (2011) 348 [arXiv: 1002.1358] [InSPIRE].

[63] M. Taronna, Higher-spin interactions: four-point functions and beyond, JHEP 04 (2012) 029 [arXiv: 1107.5843] [INSPIRE].

[64] W. Rühl, Solving Noether's equations for gauge invariant local Lagrangians of $N$ arbitrary higher even spin fields, arXiv:1108.0225 [INSPIRE].

[65] A.K.H. Bengtsson, Investigations into light-front quartic interactions for massless fields (I): non-constructibility of higher spin quartic Amplitudes, JHEP 12 (2016) 134 [arXiv: 1607.06659] [INSPIRE].

[66] M. Taronna, On the non-local obstruction to interacting higher spins in flat space, JHEP 05 (2017) 026 [arXiv: 1701.05772] [rNSPIRE].

[67] R. Roiban and A.A. Tseytlin, On four-point interactions in massless higher spin theory in flat space, JHEP 04 (2017) 139 [arXiv: 1701.05773] [INSPIRE].

[68] D. Ponomarev, A note on (non)-locality in holographic higher spin theories, Universe 4 (2018) 2 [arXiv: 1710.00403] [inSPIRE].

[69] A. Campoleoni, S. Fredenhagen, S. Pfenninger and S. Theisen, Asymptotic symmetries of three-dimensional gravity coupled to higher-spin fields, JHEP 11 (2010) 007 [arXiv: 1008.4744] [INSPIRE].

[70] A. Campoleoni, S. Fredenhagen, S. Pfenninger and S. Theisen, Towards metric-like higher-spin gauge theories in three dimensions, J. Phys. A 46 (2013) 214017 [arXiv:1208.1851] [INSPIRE].

[71] S. Fredenhagen and P. Kessel, Metric- and frame-like higher-spin gauge theories in three dimensions, J. Phys. A 48 (2015) 035402 [arXiv:1408.2712] [INSPIRE].

[72] S. Gwak, E. Joung, K. Mkrtchyan and S.-J. Rey, Rainbow valley of colored (Anti) de Sitter gravity in three dimensions, JHEP 04 (2016) 055 [arXiv: 1511.05220] [INSPIRE].

[73] S. Gwak, E. Joung, K. Mkrtchyan and S.-J. Rey, Rainbow vacua of colored higher-spin (A)dS 3 gravity, JHEP 05 (2016) 150 [arXiv:1511.05975] [INSPIRE].

[74] A. Campoleoni, S. Fredenhagen and J. Raeymaekers, Quantizing higher-spin gravity in free-field variables, JHEP 02 (2018) 126 [arXiv: 1712.08078] [INSPIRE].

[75] P. Benincasa and E. Conde, Exploring the S-matrix of massless particles, Phys. Rev. D 86 (2012) 025007 [arXiv: 1108.3078] [INSPIRE].

[76] D. Ponomarev, Off-shell spinor-helicity amplitudes from light-cone deformation procedure, JHEP 12 (2016) 117 [arXiv:1611.00361] [INSPIRE].

[77] B. Nagaraj and D. Ponomarev, Spinor-helicity formalism for massless Fields in AdS 4 , Phys. Rev. Lett. 122 (2019) 101602 [arXiv:1811.08438] [INSPIRE].

[78] B. Nagaraj and D. Ponomarev, Spinor-helicity formalism for massless fields in AdS II: $_{4}$ potentials, arXiv:1912.07494 [INSPIRE].

[79] D. Ponomarev and E.D. Skvortsov, Light-front higher-spin theories in flat space, J. Phys. A 50 (2017) 095401 [arXiv: 1609.04655] [INSPIRE].

[80] R.R. Metsaev, Light-cone gauge cubic interaction vertices for massless fields in AdS $S_{4}$, Nucl. Phys. B 936 (2018) 320 [arXiv:1807.07542] [INSPIRE]. 
[81] R.R. Metsaev, Cubic interactions for arbitrary spin $\mathcal{N}$-extended massless supermultiplets in 4d flat space, JHEP 11 (2019) 084 [arXiv:1909.05241] [InSPIRE].

[82] D. Ponomarev, Chiral higher spin theories and self-Duality, JHEP 12 (2017) 141 [arXiv: 1710.00270] [INSPIRE].

[83] M. Fierz, Über die relativistische Theorie kräftefreier Teilchen mit beliebigem Spin, Helv. Phys. Acta 12 (1939) 297.

[84] N. Boulanger, T. Damour, L. Gualtieri and M. Henneaux, Inconsistency of interacting, multigraviton theories, Nucl. Phys. B 597 (2001) 127 [hep-th/0007220] [INSPIRE].

[85] X. Bekaert, N. Boulanger and S. Cnockaert, Spin three gauge theory revisited, JHEP 01 (2006) 052 [hep-th/0508048] [INSPIRE].

[86] B. de Wit and D.Z. Freedman, Systematics of higher spin gauge fields, Phys. Rev. D 21 (1980) 358 [INSPIRE].

[87] R. Manvelyan, K. Mkrtchyan, W. Rühl and M. Tovmasyan, On nonlinear higher spin curvature, Phys. Lett. B 699 (2011) 187 [arXiv:1102.0306] [INSPIRE].

[88] R. Manvelyan and W. Rühl, The generalized curvature and Christoffel symbols for a higher spin potential in $A d S_{(}(d+1)$ space, Nucl. Phys. B 797 (2008) 371 [arXiv:0705.3528] [INSPIRE].

[89] M.S. Costa, J. Penedones, D. Poland and S. Rychkov, Spinning conformal correlators, JHEP 11 (2011) 071 [arXiv:1107.3554] [InSPIRE].

[90] S. Giombi, S. Prakash and X. Yin, A note on CFT correlators in three dimensions, JHEP 07 (2013) 105 [arXiv: 1104.4317] [INSPIRE].

[91] S. Fredenhagen, O. Krüger and K. Mkrtchyan, Constraints for three-dimensional higher-Spin interactions and conformal correlators, Phys. Rev. D 100 (2019) 066019 [arXiv: 1812.10462] [INSPIRE].

[92] P. Kravchuk and D. Simmons-Duffin, Counting conformal correlators, JHEP 02 (2018) 096 [arXiv: 1612.08987] [INSPIRE].

[93] J.R. David, M.R. Gaberdiel and R. Gopakumar, The heat kernel on $A d S_{3}$ and its applications, JHEP 04 (2010) 125 [arXiv:0911.5085] [INSPIRE].

[94] S. Giombi and I.R. Klebanov, One loop tests of higher spin AdS/CFT, JHEP 12 (2013) 068 [arXiv:1308.2337] [INSPIRE].

[95] E. Joung and M. Taronna, A note on higher-order vertices of higher-spin fields in flat and (A) $d S$ space, arXiv:1912.12357 [INSPIRE]. 